\title{
The Tevatron Chromaticity Tracker
}

\author{
Cheng-Yang Tan \\ Accelerator Division/Tevatron
}

\begin{abstract}
The Tevatron chromaticity tracker (CT) has been successfully commissioned and is now operational. The basic idea behind the CT is that when the phase of the Tevatron RF is slowly modulated, the beam momentum is also modulated. This momentum modulation is coupled transversely via chromaticity to manifest as a phase modulation on the betatron tune. Thus by phase demodulating the betatron tune, the chromaticity can be recovered. However, for the phase demodulation to be successful, it is critical that the betatron tune be a coherent signal that can be easily picked up by a phase detector. This is easily done because the Tevatron has a phase locked loop (PLL) based tune tracker which coherently excites the beam at the the betatron tune.
\end{abstract}




\section{INTRODUCTION}

In the traditional method for measuring chromaticity, the RF frequency is changed and the excursion of the betatron tune from its nominal position is measured from which the chromaticity can be extracted with the formula

$$
\Delta Q=-\frac{\xi}{\eta}\left(\frac{\Delta f}{f_{\mathrm{RF}}}\right)
$$

where $\xi$ is the chromaticity, $\eta$ is the slip factor, $f_{\mathrm{RF}}$ is the nominal RF frequency, $\Delta f$ is the change in RF frequency from $f_{\mathrm{RF}}$ and $\Delta Q$ is the change in betatron tune from the nominal betatron tune when $\Delta f=0$. It is obvious from (1) that if there is a way to continuously track the tune, a slow frequency modulation of the RF will allow the continuous measurement of chromaticity. In fact, this technique has been applied successfully at both RHIC (Relativistic Heavy Ion Collider) and at the SPS (Super Protron Synchrotron), ${ }^{\dagger}$ because both machines have phase locked loop (PLL) tune trackers which measure the betatron tunes continuously with high precision.

We have performed the above technique at the Tevatron with our PLL tune tracker. ${ }^{1}$ The results, however, have been mixed. This technique works with uncoalesced beam, but for coalesced beam, it always gives a smaller chromaticity value than expected. ${ }^{2}$ We decided to pursue the phase modulation technique first proposed by D. McGinnis ${ }^{3}$ to see if the incorrect chromaticity measurements with coalesced beam can be mitigated. $\ddagger$ And if this technique can be demonstrated to work, it can be added to the arsenal of chromaticity measurement techniques. An advantage of the phase modulation technique is that when the frequency of the phase modulation is chosen so that it lies outside the tune tracker PLL loop bandwidth, the TT is not stressed because it does not "see" the modulation and

$\dagger$ This will also be the baseline technique for the LHC (Large Hadron Collider)

$\ddagger$ In fact, this method also shows a smaller value of chromaticity for coalesced beam. We suspect that this is due to the difference in transverse emittance between coalesced and uncoalesced beam. This will be discussed in this paper. 
thus will not track it. This is unlike the traditional technique where the TT tracks the tune motion from the RF frequency changes.

In this paper, we will summarise the theory behind the phase modulation method and discuss the problems we have found in our first attempt to implement it and how we have solved them. We will also show the measurements with beam which demonstrates the performance of the CT. Finally, we will show some reasons why the chromaticity measured with the $\mathrm{CT}$ or with the traditional method with the TT for coalesced beam is smaller than we expect. 


\section{THEORY}

The theory has been worked out in other papers ${ }^{3,4}$ and we will not repeat it here. We quote the relevant formula here which relates the phase amplitude of the phase demodulated signal to chromaticity for betatron mode $(k, \pm)$

$$
Z_{ \pm}=\left(k \pm Q_{0} \mp \frac{\xi}{\eta}\right) \frac{\Delta \phi_{\bmod }}{h}
$$

where $k \in \mathbb{N} \cup\{0\}$ is the mode number, $Q_{0}$ is the fractional betatron tune, $\xi$ is the chromaticity, $\eta$ is the slip factor, $\Delta \phi_{\text {mod }}$ is the amplitude of the phase modulation applied to the accelerating $\mathrm{RF}, h$ is the harmonic number and $Z_{ \pm} \in \mathbb{R}$ is the amplitude of the phase demodulated signal. Solving for $\xi$, we have

$$
\xi_{ \pm}=\eta\left( \pm k+Q_{0} \mp \frac{h Z_{ \pm}}{\Delta \phi_{\bmod }}\right)
$$

In particular, for the Tevatron, the $\mathrm{CT}$ looks at the $(k,+)$ mode, $\$$ i.e.

$$
\xi_{+}=\eta\left[\left(k+Q_{0}\right)-\frac{h Z_{+}}{\Delta \phi_{\bmod }}\right]
$$

Therefore, it is obvious from (4) that once we can measure the phase amplitude $Z_{+}$and the betatron tune $Q_{0}$, the chromaticity $\xi$ is easily calculated. The parameters which are relevant to the Tevatron and CT are shown in Table 1.

In practice, the measurement of the phase oscillation w.r.t. the betatron tune is difficult to accomplish if the betatron tune is not coherent. In fact, early experiments performed by D. McGinnis using this technique did not yield satisfactory results because the betatron tune from the Schottky pickups is an incoherent signal and therefore the phase is not well defined. It was not until the TT system became operational that this technique became feasible because the TT excites the beam coherently. And thus the phase can be reliably measured w.r.t. the coherently excited betatron tune.

$\S$ In earlier papers, we have mistakenly used mode $(k,-)$. 


\begin{tabular}{|c|c|c|}
\hline Parameter & Value & Description \\
\hline$\eta$ & 0.0029 & slip factor \\
\hline$k$ & 448 & mode number \\
\hline$Q_{0}$ & $0.55-0.6$ & fractional betatron tune \\
\hline$h$ & 1113 & harmonic number \\
\hline$\Delta \phi_{\bmod }$ & $2.8^{\circ}-11.2^{\circ}$ & amplitude of phase modulation \\
\hline$\Omega_{\bmod }$ & $2 \pi \times 23 \mathrm{~s}^{-1}$ & phase modulation frequency \\
\hline$\Omega_{s}$ & $2 \pi \times(35-84) \mathrm{s}^{-1}$ & synchrotron frequency \\
\hline$\omega_{\mathrm{RF}}$ & $2 \pi \times\left(53.1 \times 10^{6}\right) \mathrm{s}^{-1}$ & RF frequency \\
\hline$\xi$ & $4-15$ & Tevatron chromaticity range \\
\hline
\end{tabular}

RF Frequency and Betatron Tune Excursion

The maximum change in betatron tune $\Delta Q_{\max }$ due to the phase modulation is given by the following formula (for derivation see Ref. 4)

$$
\Delta Q_{\max }=\left|\frac{\Delta \phi_{\bmod } \times \Omega_{\bmod }}{\omega_{\mathrm{RF}}}\left(Q_{0}-\frac{\xi}{\eta}\right)\right|
$$

And for $\Delta \phi_{\text {mod }}=10^{\circ}, Q_{0}=0.583$ plus the numbers from Table 1, we have

$$
1 \times 10^{-4}<\Delta Q_{\max }<3 \times 10^{-4} \quad 4 \leq \xi \leq 10
$$

When we translate these numbers to the traditional method which uses (1) for calculating $\xi$, we find that with the above $\Delta Q_{\max }$ excursion, the equivalent change in $\mathrm{RF}$ frequency $\Delta f$ is

$$
(1.5<\Delta f<4)_{\max } \mathrm{Hz} \quad 4 \leq \xi \leq 10
$$

We can compare $\Delta f$ found above to the traditional method when done by hand which is $\pm 40 \mathrm{~Hz}$. This means that the maximum $\Delta f$ change using the $\mathrm{CT}$ is about $10 \times$ smaller than the traditional method. 


\section{PROBLEMS}

Our early attempts with this method did not produce a chromaticity measurement better than \pm 1 unit when we measure the horizontal chromaticity. See Figure 1 . We have traced the source of the problem to the following:

(i) The vertical tune $Q_{v}$. This causes beats with $Q_{h}$. See Figure 2 .

(ii) The lower sideband of the betatron tune $\left(1-Q_{h}\right)$ adds shoulders to the input of the CT. See Figure 3.

(iii) The synchrotron frequency $Q_{s}$ which interferes with the phase demodulation.

In fact, all the above can be easily reproduced on the bench with the setup shown in Figure 4 using the parameters shown in Table 2.

\section{Beam Simulator}

The beam simulator consists of three signal generators (See Figure 4). The HP8657A is used to synchronise two signal generators HP8904 and HP33250A. The HP8904 OUT 1 is set to produce a carrier frequency which is at the horizontal betatron tune. The carrier is phase modulated by both the CT RF modulation frequency which is at $23 \mathrm{~Hz}$ and the synchrotron frequency which can range from 35 to $84 \mathrm{~Hz}$. OUT 2 is set to produce the vertical betatron frequency and summed with OUT 1 and sent to the input of the CT. The HP33250A outputs a sine wave at the horizontal betatron tune which is the reference frequency for the CT. With the settings shown in Table 2, we can reproduce the poor tracking of uncoalesced beam shown in Figure 5 . 


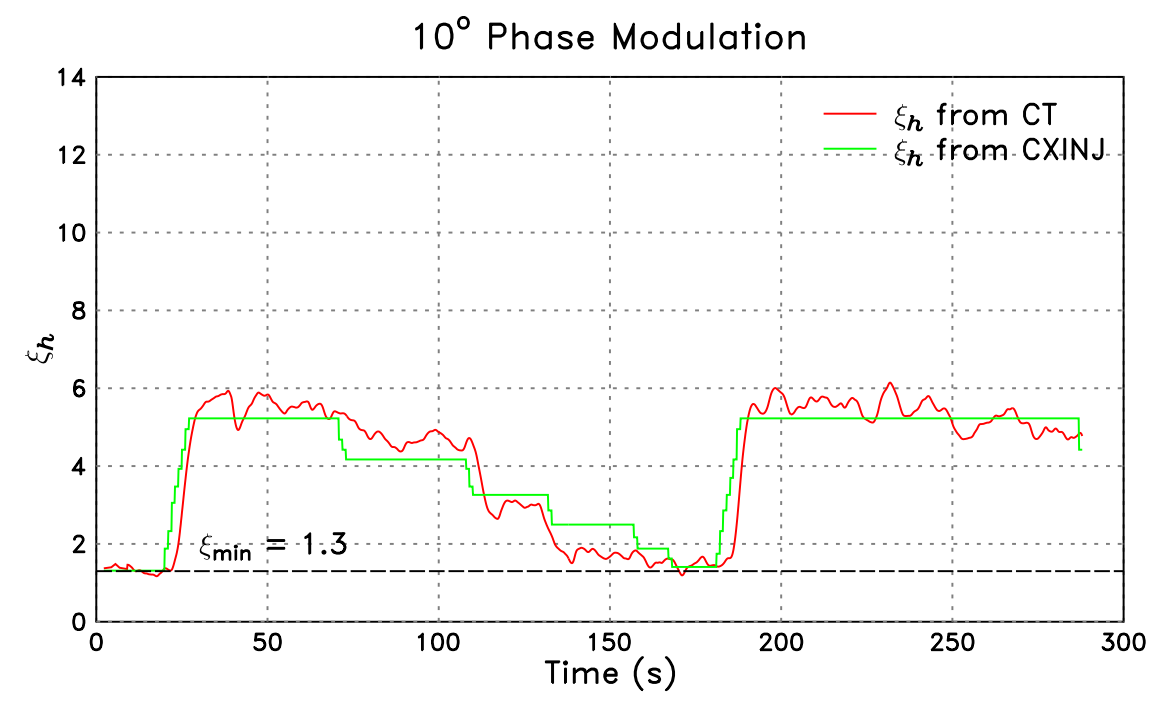

Figure 1 As the chromaticity is changed with a calibrated knob CXINJ (green), CT (red) tracks those changes but with errors as large as 1 unit of chromaticity.

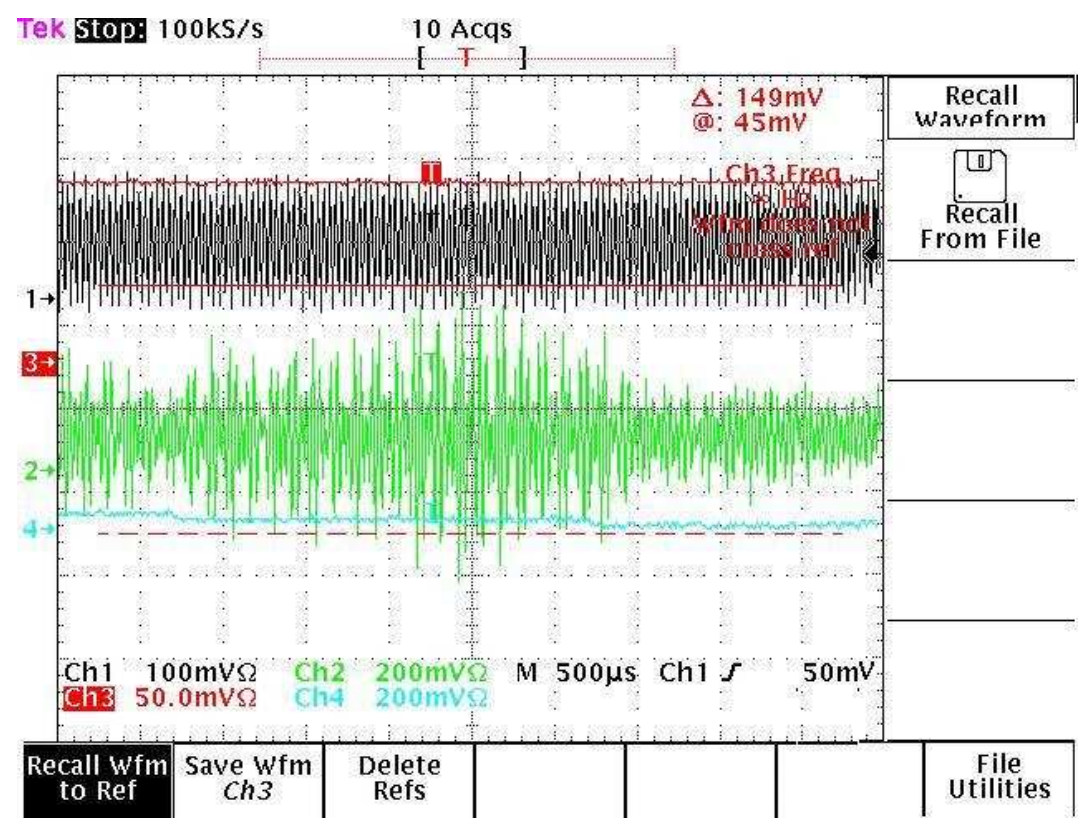

Figure 2 The green trace shows $Q_{v}$ beating with $Q_{h}$. 

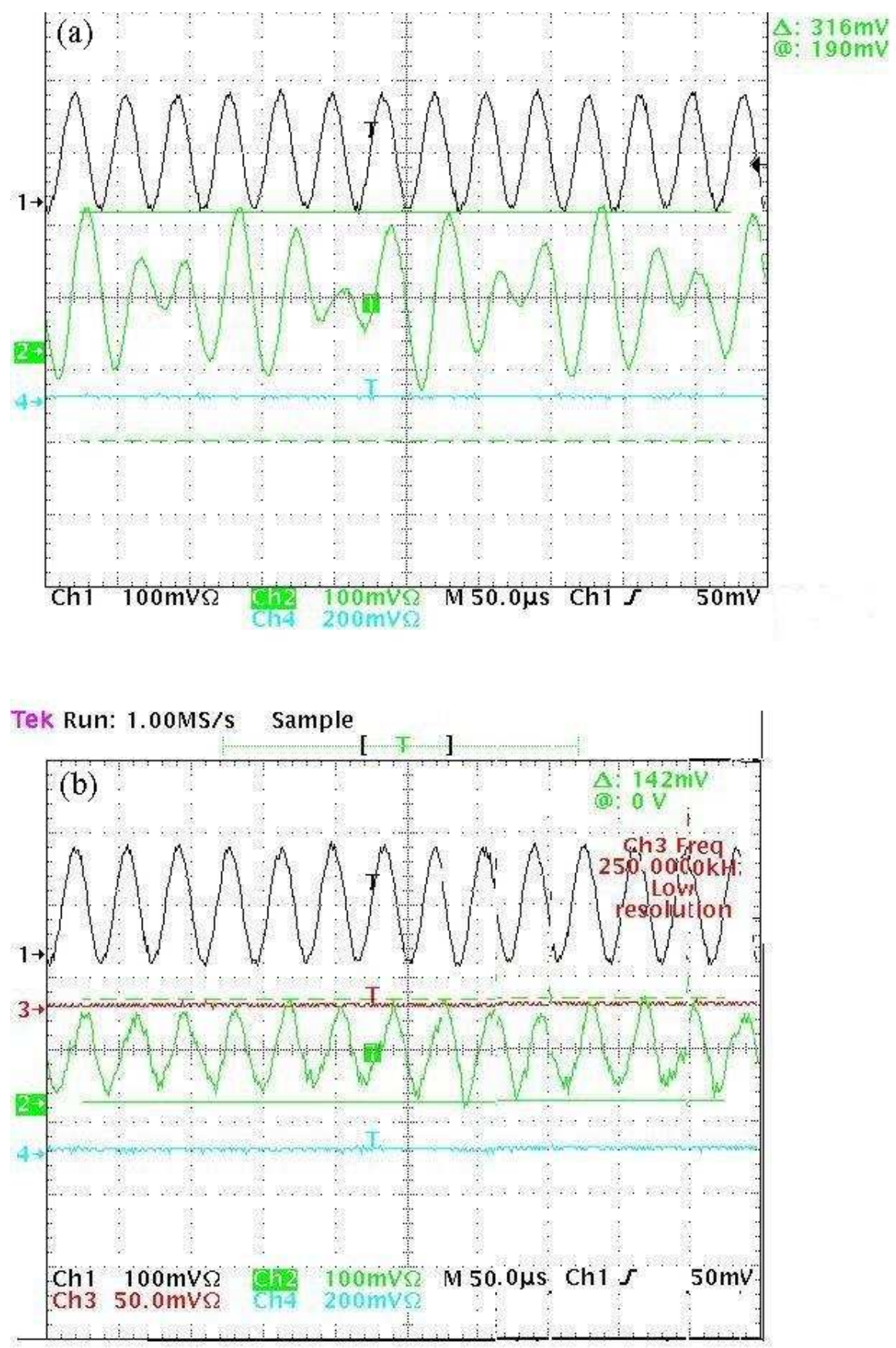

Figure 3 The shoulders shown in the green trace in (a) come from the addition of the betatron tunes $Q_{h}$ and $\left(1-Q_{h}\right)$. (b) When a high pass filter is used to suppress $\left(1-Q_{h}\right)$ and pass only $Q_{h}$, the shoulders go away. This data comes from uncoalesced beam being phase modulated at an amplitude of $5.6^{\circ}$. 


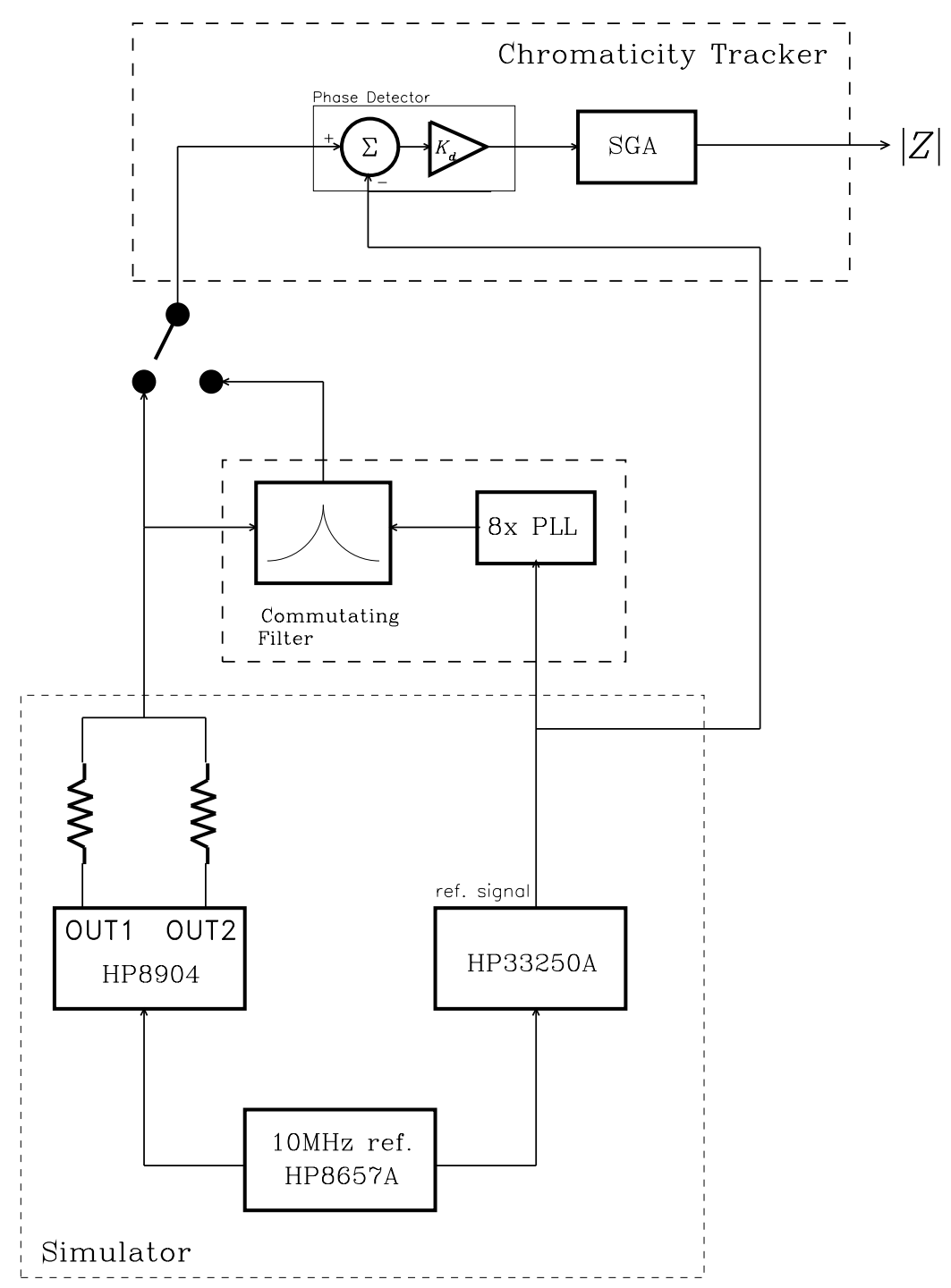

Figure 4 We can reproduce the behaviour of the $\mathrm{CT}$ with \pm 1 unit of error (See Figure 5) when the commutating filter is not in the circuit. 
Table 2. Simulator Settings

\begin{tabular}{|c|c|c|c|c|}
\hline Channel & Frequency & Amplitude & Mode & Description \\
\hline $\mathrm{A}$ & $28.1 \mathrm{kHz}$ & $200 \mathrm{mV}$ & sine & horizontal betatron freq. \\
\hline $\mathrm{B}$ & $23 \mathrm{~Hz}$ & $5^{\circ}$ & phase mod. & phase modulation \\
\hline $\mathrm{C}$ & $84 \mathrm{~Hz}$ & $45^{\circ}$ & phase mod. & synchrotron modulation \\
\hline \multicolumn{5}{|c|}{ HP8904 OUT 2 Settings } \\
\hline $\mathrm{D}$ & $27.3 \mathrm{kHz}$ & $100 \mathrm{mV}$ & sine & vertical betatron freq. \\
\hline \multicolumn{5}{|c|}{ HP33250A Settings } \\
\hline 1 & $28.1 \mathrm{kHz}$ & $200 \mathrm{mV}_{\mathrm{pp}}$ & sine & $\begin{array}{l}\text { horizontal betatron freq. } \\
\text { as reference for CT }\end{array}$ \\
\hline
\end{tabular}

Commutating Filter

Our solution for increasing the accuracy of the $\mathrm{CT}$ is to have a narrow band filter (NBF) which can filter out all the extraneous signals outside the betatron tune. However, the resonance of the NBF must dynamically track the motion of the betatron tune because it is not stationary. An NBF which can easily change its resonant frequency exists and is called a commutating filter.

The commutating filter is a series of narrow band filters whose resonances are dictated by the switching frequency $\omega_{s w}$. See Figure 6 . For a commutating filter which has $N$ identical capacitors, the resonances $\Omega_{\text {res }}$ are at

$$
\Omega_{\mathrm{res}}=k\left(\frac{\omega_{s w}}{N}\right) \quad k \in \mathbb{Z}
$$

and the bandwidth of each resonance is

$$
f_{\mathrm{bw}}=\frac{1}{\pi N R C}
$$




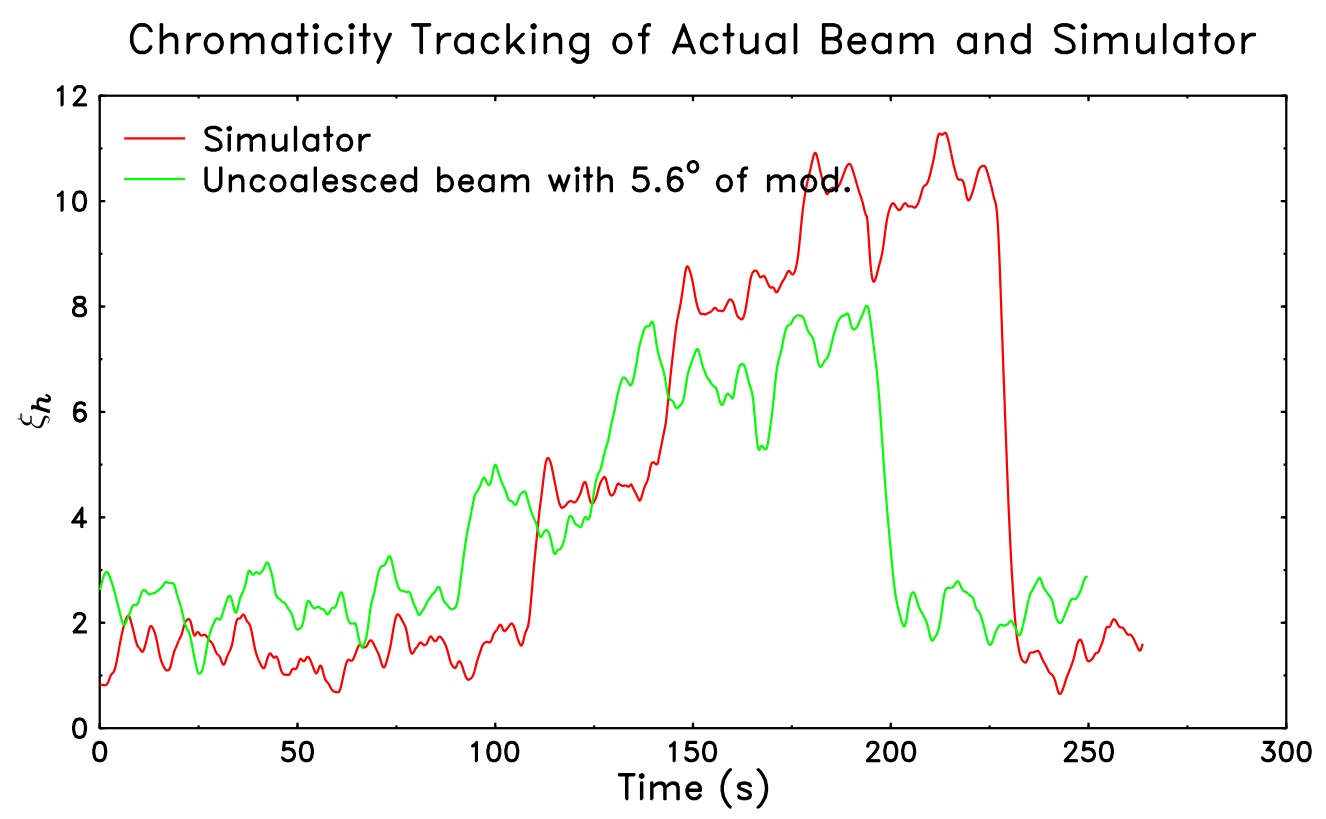

Chromaticity Tracking of Simulator with Commutating Filter Added

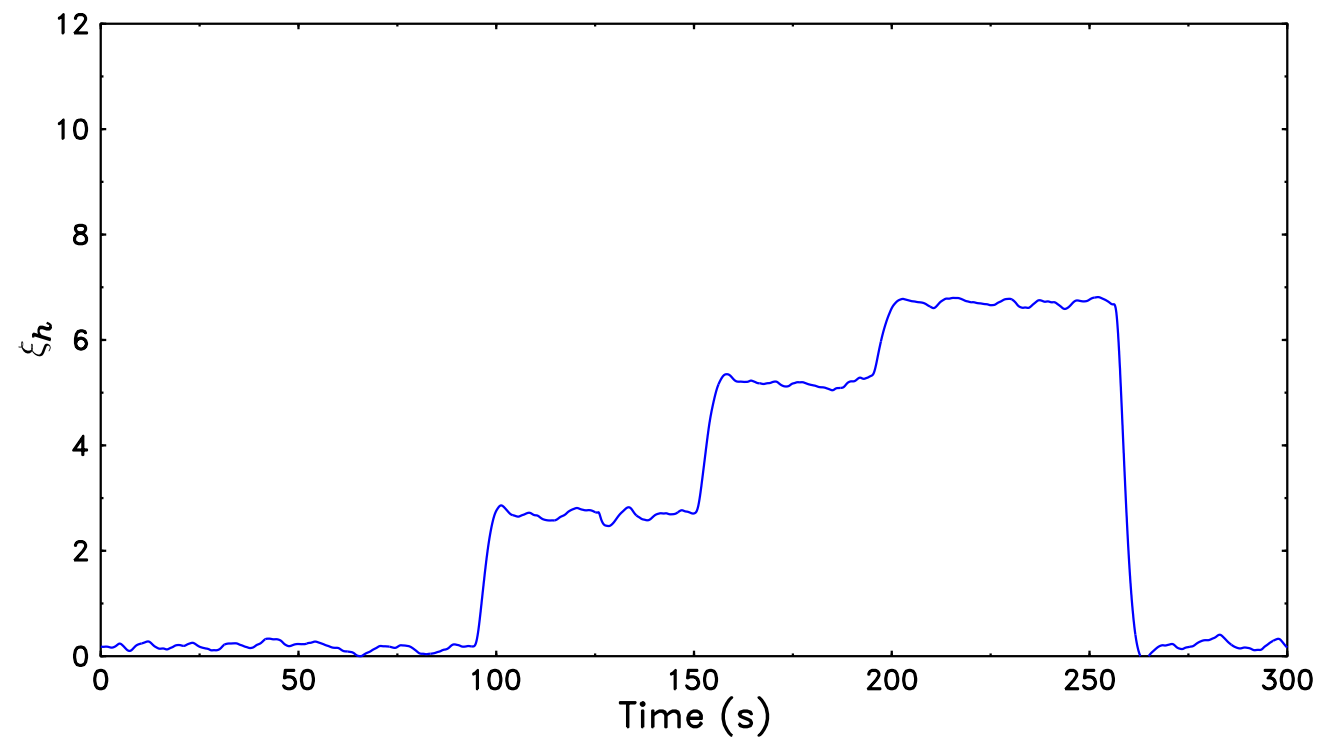

Figure 5 This shows the behaviour of the CT when tracking actual uncoalesced beam and the simulator. The settings of the simulator are shown in Table 2. It is clear that the CT tracks the simulator as poorly as actual beam when the commutating filter is not in the circuit. With the addition of the commutating filter into the circuit, it magically cleans up the noise sources and the CT can now track to \pm 0.1 units of chromaticity on the bench. 

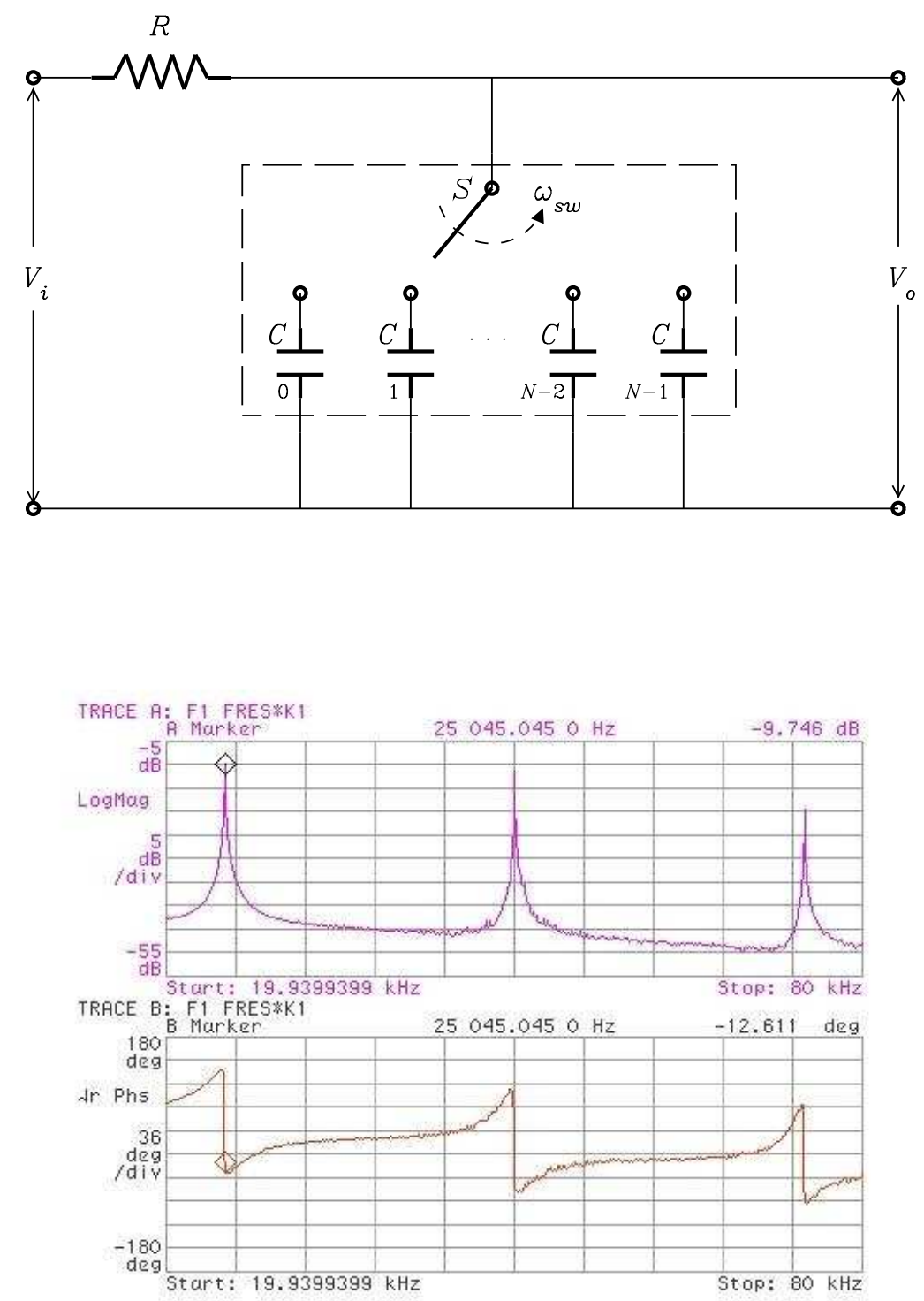

Figure 6 The commutating filter consists of a resistor $R$ and $N$ capacitors $C$ numbered from $0,1, \ldots, N-1$. The switch $S$ rotates at frequency $\omega_{s w}$ and connects each capacitor $C$ for a time period of $2 \pi / N \omega_{s w}$. The frequency response of this commutating filter is measured with a vector signal analyser (VSA) for $R=10 \mathrm{k} \Omega$ and $C=0.1 \mu \mathrm{F}$. 
where $R$ is the resistance and $C$ is the capacitance of the filter.

Therefore, when $\omega_{s w}=N \times Q_{0} \omega_{\mathrm{rev}}$, one of the resonances will lie directly on top of the betatron tune. Thus, any signals outside the resonance will be filtered out.

When this is added to the simulator at the location shown in Figure 4, we can see that tracking improves dramatically on the bench. See Figure 5. In fact, with the filter, the improvement of the CT when measuring actual beam is improved just as much. See Figure 7.

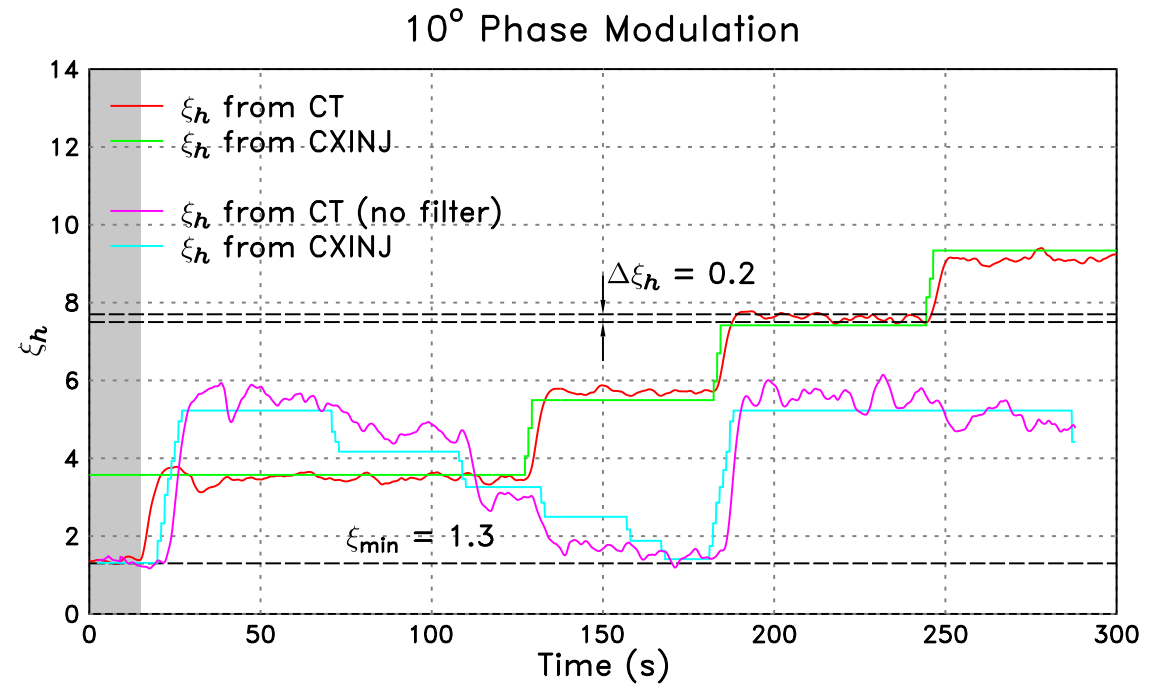

Figure 7 The data from Figure 1 is superimposed here to show the improvement in the CT with the commutating filter when measuring the chromaticity of the beam. 


\section{IMPLEMENTATION}

The current implementation of the $\mathrm{CT}$ is shown in Figure 8. At House F0, a phase modulator is connected directly to the low level RF system so that the Tevatron RF can be phase modulated. We have chosen the modulation frequency $\Omega_{\bmod }$ of the RF to be $(2 \pi \times 23) \mathrm{s}^{-1}$. The choice is dictated by the closed loop bandwidth of the PLL tune tracker which is about $5 \mathrm{~Hz}$ and the range of synchrotron frequencies of the Tevatron. See Table 1.

At House A1, our CT module picks up and processes the phase modulated betatron signal from the Tevatron 21.4 MHz Schottky system after it has been filtered with the commutating filter. At the heart of the $\mathrm{CT}$ module is the hardware phase detector (PD) which has been implemented around an ALTERA Cyclone FPGA. See Figure 9. We have designed the CT module to be compatible with both the modulation frequency and the betatron frequency. In the ALTERA, there are two major blocks:

(i) A hardware PD which extracts out the sine and cosine of the phase w.r.t. betatron tune. The inputs to the PD are the phase modulated betatron tune from the 21.4 MHz Schottky after it has been filtered with the commutating filter and the betatron tune from the TT which is the carrier frequency. These two signals are sampled at $250 \mathrm{kHz}$ which is $\sim 10 \times$ higher than the betatron frequency.

(ii) A 32-bit NIOS II floating point processor running at $50 \mathrm{MHz}$. It takes the arctangent of the sine and cosine of the phase from the hardware PD. This is done at $(16 \times 23) \mathrm{Hz}$ which yields an oscillating signal which contains the $23 \mathrm{~Hz}$ component. The amplitude of the $23 \mathrm{~Hz}$ oscillation is recovered by putting it through a Sliding Goertzel filter ${ }^{4}$ which is a clever discrete Fourier transform. In particular, we are applying a 320 point Fourier transform. The amplitude of the $23 \mathrm{~Hz}$ component is communicated back to the control system via ethernet. 
(iii) From the amplitude of the $23 \mathrm{~Hz}$ component, the chromaticity is calculated using (4) by a dæmon running on the control system.

We will give detailed specifics of the implementation in the following subsections.

\section{Hardware PD}

The hardware PD performs a series of simple trigonometric manipulations to extract out the phase component $\phi$ from the phase modulated input signal $A \sin \left(\omega_{\beta} t+\phi\right)$ w.r.t the unmodulated reference betatron signal $B \sin \omega_{\beta} t$. Both $A$ and $B$ are the amplitudes of the phase modulated and reference signals respectively. See Figure 10. The key part in the hardware PD is the Hilbert transformer. A Hilbert transformer is a type of filter which phase shifts all signals within its operational bandwidth by exactly $\pi / 2$, i.e. $\sin (\omega t+$ $\phi) \stackrel{\text { Hilbert }}{\longrightarrow} \cos (\omega t+\phi)$. In particular, our Hilbert transformer is a 51 tap finite impulse response (FIR) filter. See Appendix II. A 51 tap FIR filter has a 25 tap delay, which is why delays are required in the hardware PD.

When we write out the mathematics of the block diagram shown in Figure 10, we find that the output of the hardware PD is exactly $A B \sin \phi$ and $A B \cos \phi$. Note: it is necessary that there is no DC offset in either the modulated betatron signal or the reference signal. Any DC offset will give an undesirable oscillation at $\omega_{\beta}$ at the output.

\section{Calculating $\phi$}

Once the hardware PD provides us with $A B \sin \phi$ and $A B \cos \phi$, it is obvious that we have to take the arctangent of $\sin \phi / \cos \phi$ to obtain $\phi$. However, the arctangent is a multiple-valued function and in all standard implementations of the arctangent in $\mathrm{C}$ or 


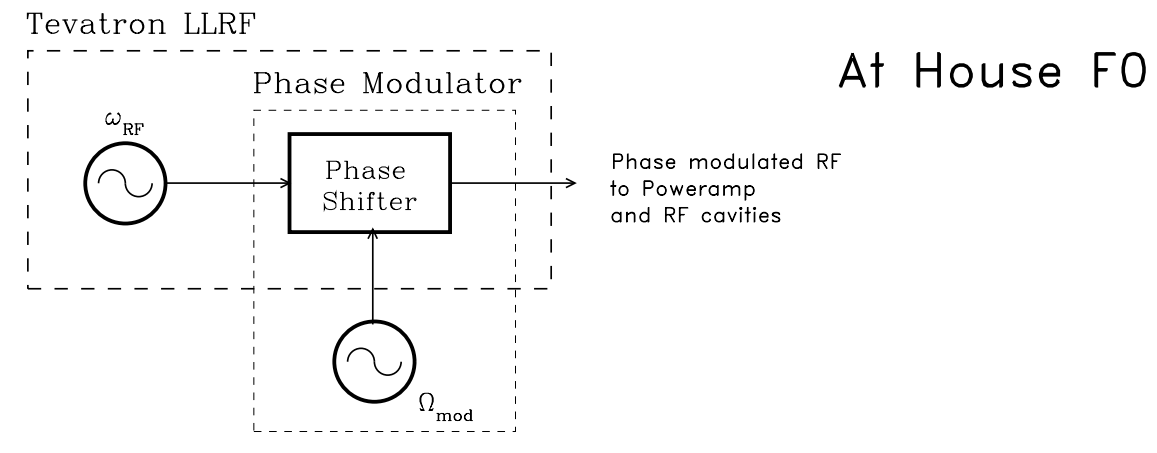

\section{At House A1}

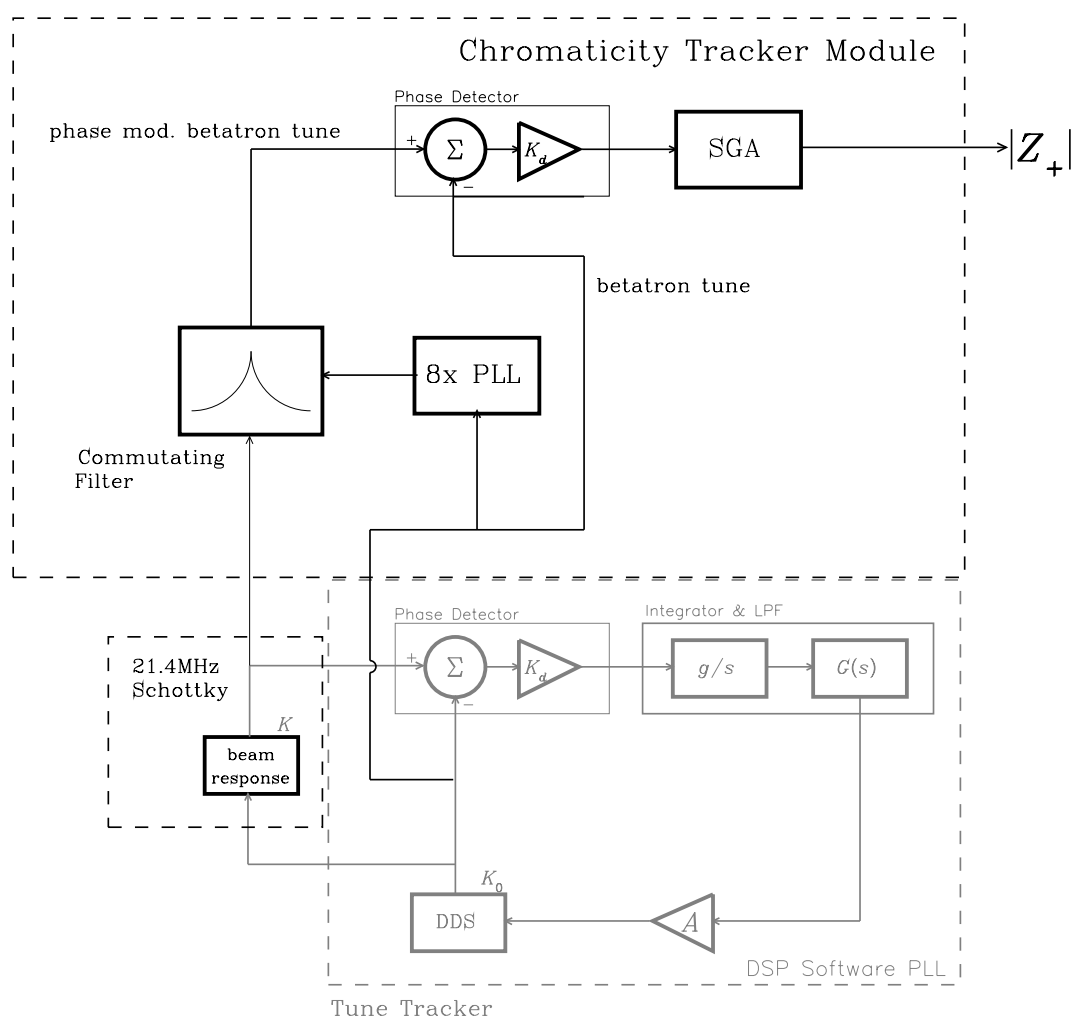

Figure 8 The current implementation of the CT consists of a phase modulator at House F0 which phase modulates the Tevatron RF. At House A1, the betatron signal is tracked and excited by the tune tracker PLL. The phase locked signal from the direct digital synthesiser (DDS) of the tune tracker PLL is used as the reference signal for the phase detector of the CT. It is also upconverted $8 \times$ for the commutating filter. 
FORTRAN, the arctangent of vectors like $(-1, \epsilon)$ and $(-1,-\epsilon)$ where $\epsilon \ll 1$ return

$$
\left.\begin{array}{c}
\arctan (-1, \epsilon)=\pi \\
\arctan (-1,-\epsilon)=-\pi
\end{array}\right\}
$$

where $\arctan (x, y)$ is the arctangent of the vector $(x, y)$ in the $x y$-plane. 9

For example, if we have $\varphi$ oscillating about $-\pi$ as follows

$$
\varphi(t)=-\pi+\sin 2 \pi t
$$

then when we use the usual implementation of arctangent defined above on the vector $(\cos \varphi, \sin \varphi)$, we find that the result is discontinuous because of the arguments we gave above. See Figure 11.

The solution to the problem is trivial. We simply increment a counter called the winding number $n$ when the vector $(\cos \varphi, \sin \varphi)$ rotates from the third quadrant to the fourth and decrement it when it rotates from the fourth to the third. Therefore, our formula which includes the winding number is

$$
\operatorname{atan} 2 u n w i n d(n, x, y)=2 n \pi+\arctan (x, y)
$$

When we apply atan2unwind() to the above example, we find that it exactly reproduces $\varphi(n)$. Again see Figure 11. A C implementation of atan2unwind() is shown in Appendix III. Note: This algorithm is the panacea if the vector $(x, y)$ rotates smoothly from quadrant to quadrant. On the other hand, if $(x, y)$ jumps from quadrant 2 to quadrant 4 , atan2unwind() will have a discontinuity.

\section{Calculating $\left|Z_{+}\right|$}

$\phi$ at the output of the phase detector is a sinusoidal function in time. In fact, we can write it as

$$
\phi(t)=Z_{+} \sin \left(\Omega_{\bmod } t+\theta\right)+\Phi(t)
$$

The standard $\mathrm{C}$ arctangent implementation has its arguments flipped, i.e. atan2(y, x). 

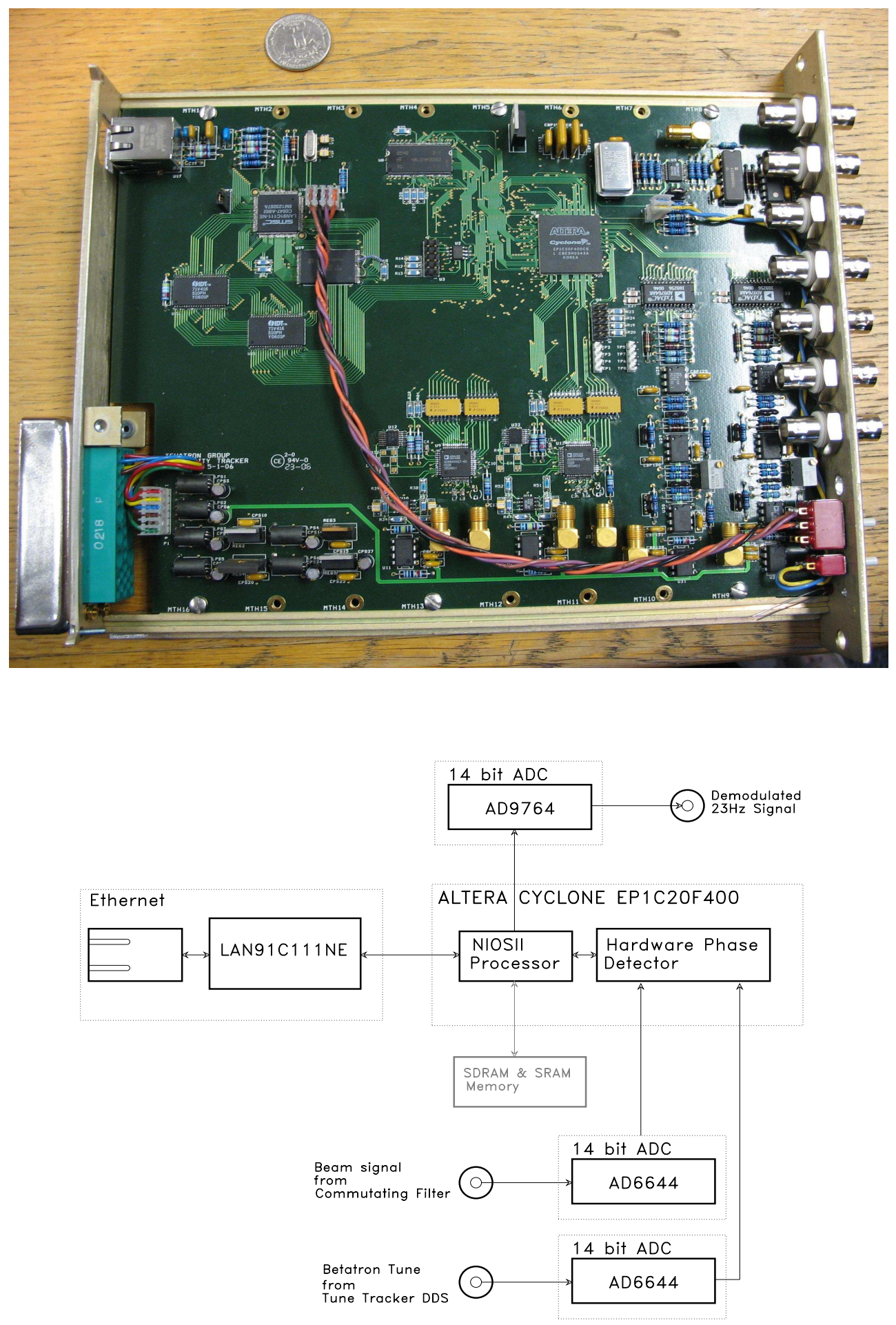

Figure 9 The CT module lives on a NIM card. A diagram of the essential blocks of the electronics on the card is also shown here. 


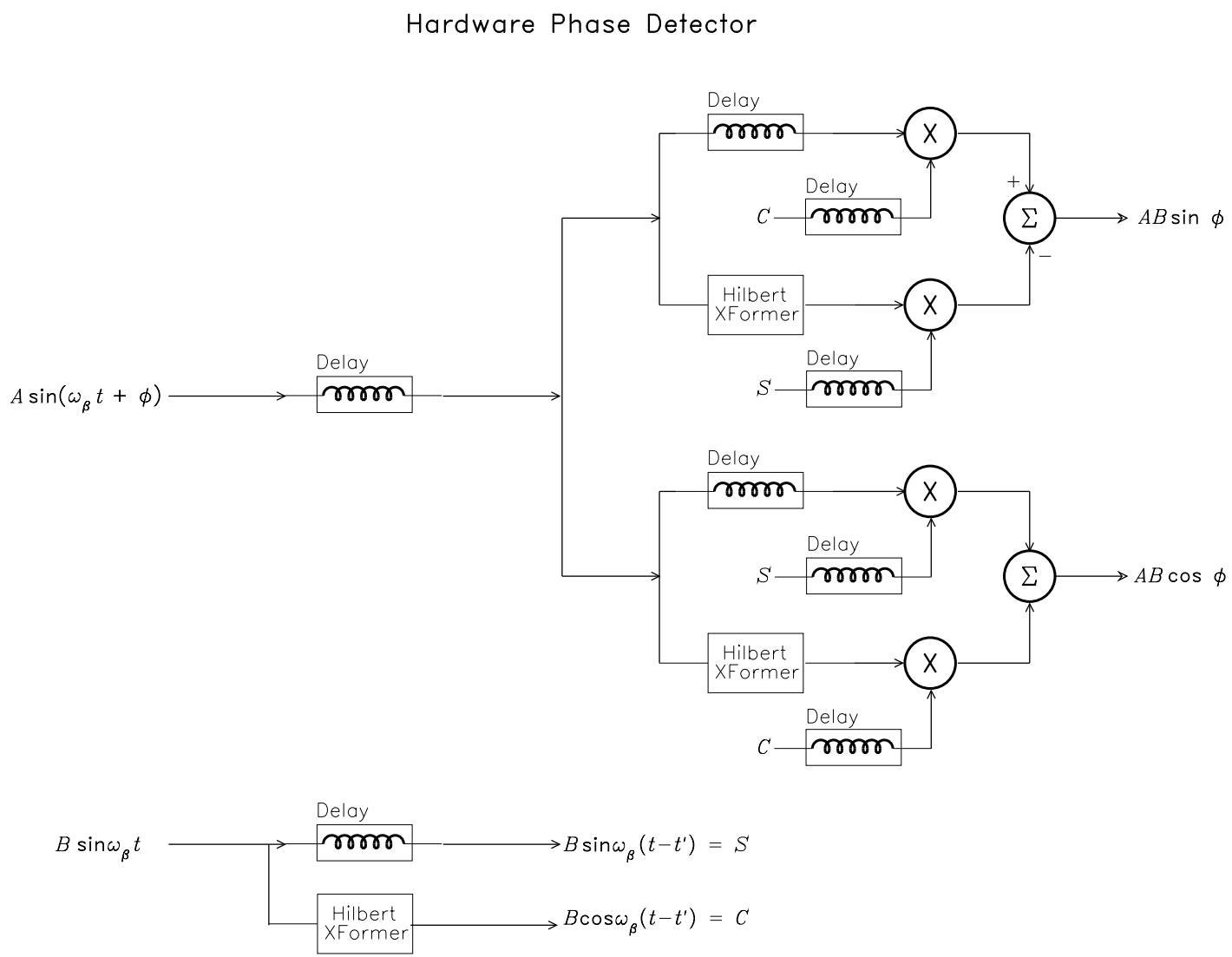

Figure 10 The hardware phase detector is implemented in an ALTERA FPGA. The phase modulated betatron signal $A \sin \left(\omega_{\beta} t+\phi\right)$ is referenced to the betatron signal $B \sin \omega_{\beta} t$ in order to extract out $\sin \phi$ and $\cos \phi$. Note: $t^{\prime}$ is a 25 tap delay and it is necessary to create $\cos \omega_{\beta}\left(t-t^{\prime}\right)$ and $\sin \omega_{\beta}\left(t-t^{\prime}\right)$ from $\sin \omega_{\beta} t$ because the TT only supplies the reference sine signal. All mathematics in the FPGA are performed in 16-bit fixed point arithmetic.

where $\theta$ is the phase w.r.t. the sampling frequency and $\Phi(t)$ contains phase oscillations from synchrotron motion and phase noise.

The usual way to extract out $Z_{+}$is to Fourier transform $\phi(t)$ and then read off the magnitude of the Fourier component at $\Omega_{\mathrm{mod}}$. In fact, since the $\phi(t)$ data stream is flowing 


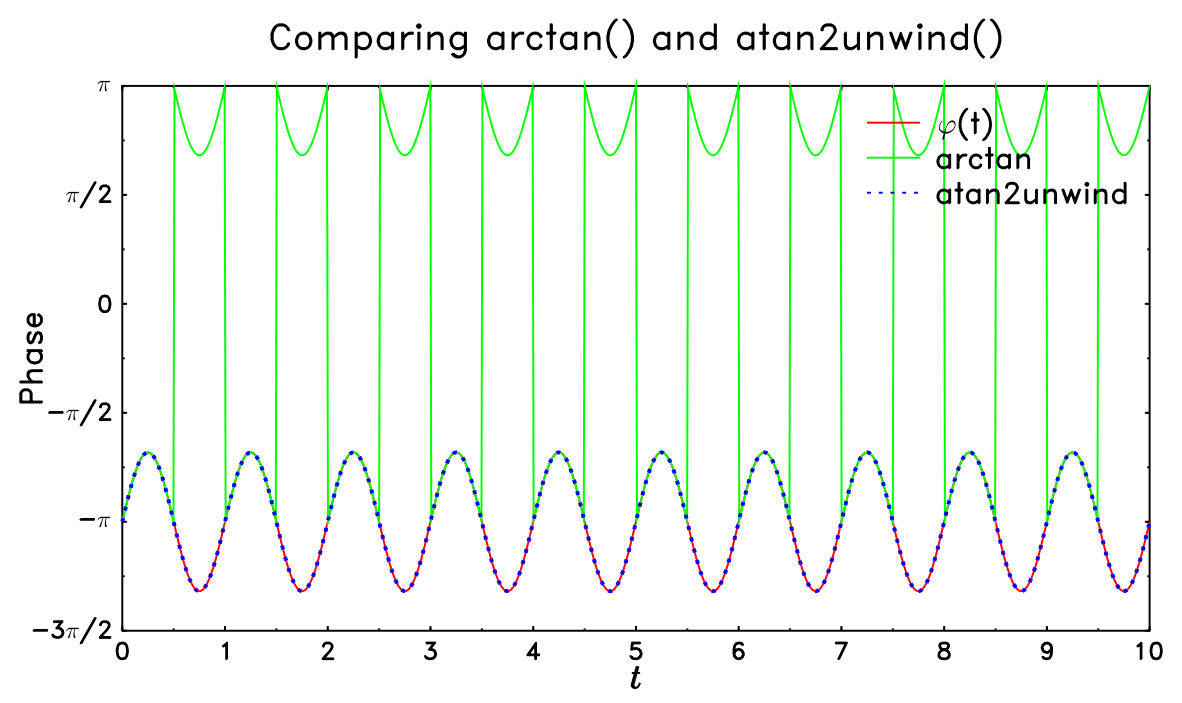

Figure 11 This graph shows the behaviour of $\arctan (\cos \varphi, \sin \varphi)$ and $\operatorname{atan} 2$ unwind $(\cos \varphi, \sin \varphi)$. Clearly, $\arctan ()$ shows discontinuities at $\pm \pi$, while atan2unwind () exactly matches $\varphi(t)$.

in continuously, we will have to continously perform FFT's. This is terribly inefficient because an $N$ point FFT calculates all $N / 2$ Fourier coefficients when we are only interested in the Fourier amplitude at $\Omega_{\bmod }$. Instead, we use a method called the Sliding Goertzel Algorithm (SGA) which is much more efficient because for $N$ equally spaced input samples, the SGA only requires $(N+1)$ multiplications and $(2 N-1)$ additions for obtaining $Z_{+}$for the first $Z_{+}$and then 3 multiplications and 5 additions for obtaining subsequent $Z_{+}$'s. In contrast, a radix-2 FFT requires $\log _{2} N+1$ multiplications and $\frac{3}{2} \log _{2} N+\frac{1}{2}$ additions for calculating every $Z_{+}$. Therefore, for $N>4$, the SGA is a more efficient algorithm than the radix-2 FFT. For a complete derivation of the SGA, see Appendix II of Ref. 4.

Once $Z_{+}$is extracted from $\phi(t), \xi$ is calculated using (4). However, there is a limitation which we will discuss in the next subsection. 


\section{Limitations}

The present CT implementation has the limitation that the phase detector only returns $\left|Z_{+}\right|$because we do not measure the phase relationship between the $23 \mathrm{~Hz}$ at the CT and the $23 \mathrm{~Hz}$ from the signal generator which is injected into the phase shifter of the LLRF. See Figure 8. This means that there will always be a sign ambiguity in $\xi$ measured by the CT. Fortunately, the Tevatron is set up to run with positive chromaticity only, and with this knowledge, we can see that $Z_{+} \leq 0$ and so (4) becomes

$$
\xi_{+}=\eta\left[\left(k+Q_{0}\right)+\frac{h\left|Z_{+}\right|}{\Delta \phi_{\bmod }}\right] \geq \xi_{\min } \quad \text { for } Z_{+} \leq 0
$$

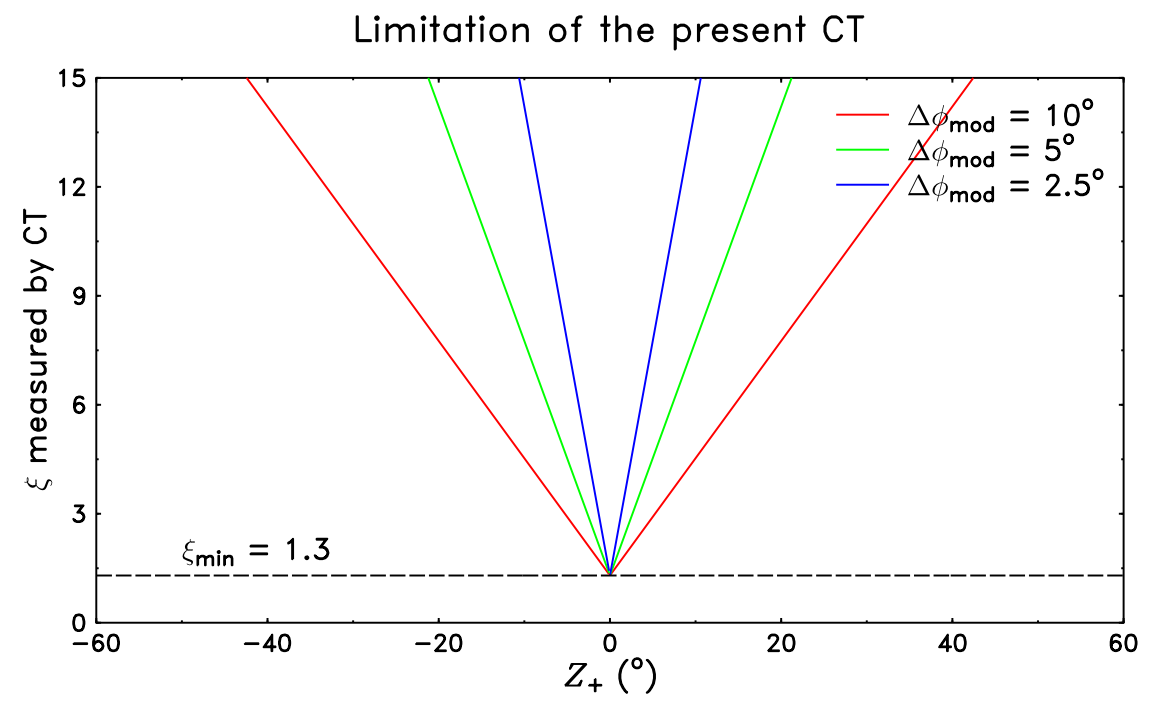

Figure 12 When we use (14) for calculating $\xi$, it is always positive because the present $\mathrm{CT}$ only measures $\left|Z_{+}\right| \geq 0$. The smallest value of $\xi$ that can be measured presently is 1.3 units.

In fact, the minimum chromaticity $\xi_{\min }$ that can be measured by the $\mathrm{CT}$ is when $Z_{+}=0$. In particular, we can use the parameters for the Tevatron and CT from Table 1 to calculate 
$\xi_{\min }$ which is

$$
\xi_{\min }=\eta\left(k+Q_{0}\right) \approx 0.0029 \times 448.575=1.3
$$

We plot the full range of $\xi$ that can be returned by the current implementation in Figure 12 . 


\section{MEASUREMENTS}

We can measure the chromaticity of uncoalesced protons with the CT as a function of the chromaticity sextupole setting CXINJ. In the Tevatron, the term "uncoalesced protons" means filling 30 adjacent RF buckets in the Tevatron with protons to give a total of $200 \times 10^{9}$ to $300 \times 10^{9}$ protons. After we have calibrated CXINJ using the traditional method by hand, we can plot the CT measured chromaticity as a function of calibrated CXINJ settings. See Figure 13.

We notice that the CT returns a much smaller value of chromaticity for $\xi_{h}>10$. This problem is not fully understood because the CT measures large chromaticities on the bench using the beam simulator described in the previous subsection Beam Simulator. Computer simulations give us some idea about why this happens but it is not a full explanation. See the section CT Large Chromaticity Limitations below.

\section{Chromaticity Drift At Injection}

The Tevatron is a superconducting machine and so the persistent currents in its dipole

magnets depend on its ramp and squeeze history. ${ }^{5}$ These persistent currents slowly decay away and one manifestation is a drift of the chromaticity at the injection porch. Figure 14(b) shows the evolution of $\xi_{h, v}$ from the decay of the persistent currents at the injection porch as a function of time. The ramp history of the Tevatron before this plot was made is as follows: the Tevatron was left at its collision energy at $980 \mathrm{GeV}$ and squeezed for 15 minutes and then unsqueezed and ramped down to its injection energy at $150 \mathrm{GeV}$.

An interesting feature in Figure 14(a) is that $\xi_{h}$ has some dependence on beam current. This observation is confirmed by both hand measurements with the traditional method as 

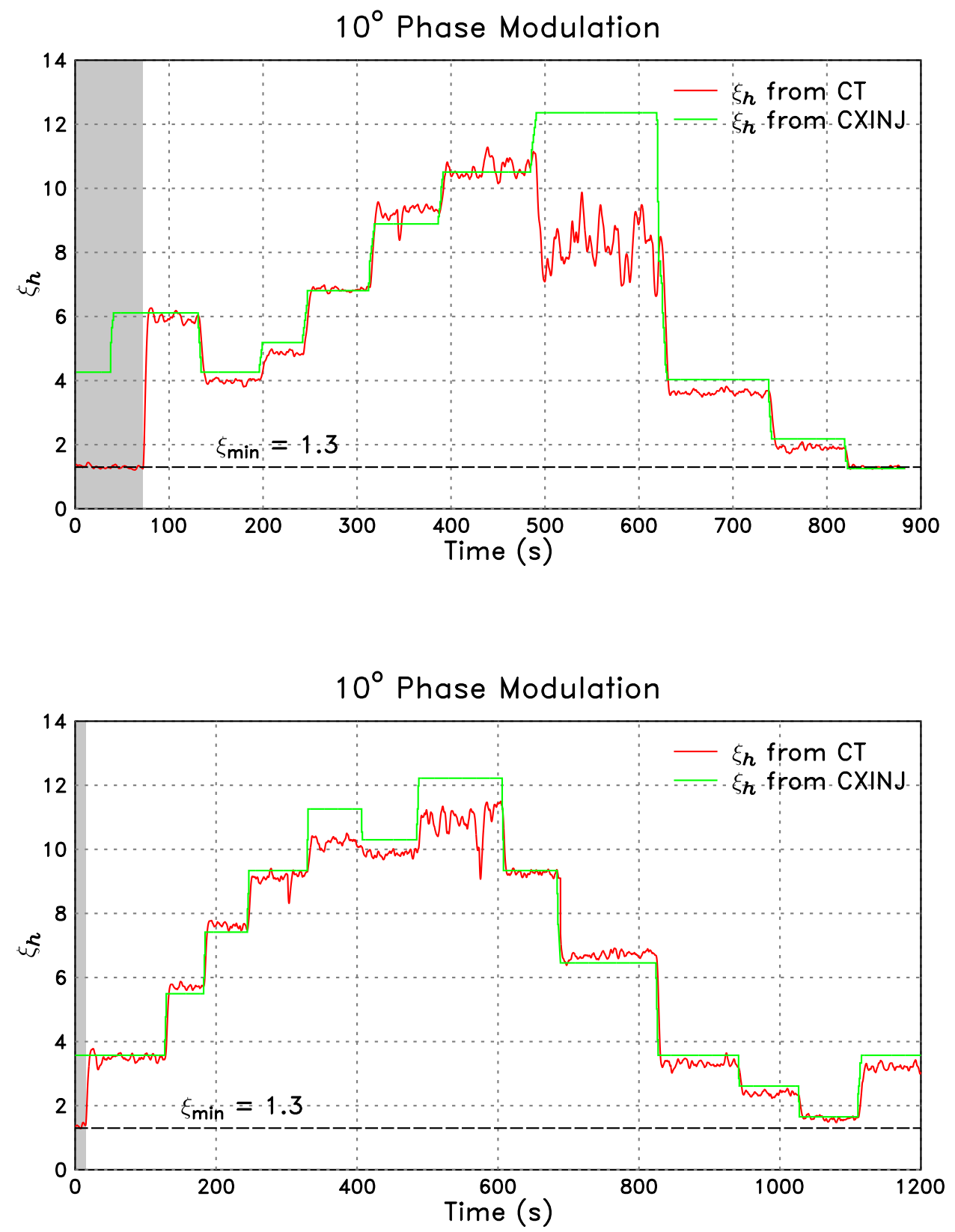

Figure 13 The grey area is when the phase modulaton is off. After it is turned on, the CT tracks the changes in chromaticity when the calibrated CXINJ is changed. Notice that when $\xi_{h}>10$, the CT returns a smaller chromaticity than the actual $\xi_{h}$. 
well as with the CT measurements. After the third injection of beam around $2500 \mathrm{~s}, \xi_{h}$ jumps from 6 units before injection to 8 units after reinjection with lower beam current.

\section{Measurements with Coalesced Proton Bunches}

Up to this point, we have been measuring chromaticity with uncoalesced protons. We now turn our attention to coalesced bunches of protons. In the Tevatron, the term "coalesced bunch" means one bucket is filled with $200 \times 10^{9}$ to $300 \times 10^{9}$ protons. In our experiment, we fill the Tevatron with 4 bunches of coalesced protons which are spaced 21 buckets apart. When we measure the chromaticity of coalesced protons with the CT (or the TT), it turns out that the CT measures a smaller value of chromaticity than what we expect. See Figure 15. The CT measures $\xi_{h} \approx 2$ and $\xi_{v} \approx 3$ while the traditional method measures $\xi_{h} \approx 4.5$ and $\xi_{v} \approx 2$. This implies a difference of $\Delta \xi_{h}=-2.5$ and $\Delta \xi_{v}=+1$ when compared to the traditional method.

\begin{tabular}{c|c|c}
\hline \multicolumn{2}{c}{ Table 4. Uncoalesced and Coalesced Beam Parameters } \\
\hline Parameter & Uncoalesced & Coalesced \\
$\sigma_{x}(\mathrm{~mm})$ & 0.88 & 1.50 \\
$\sigma_{y}(\mathrm{~mm})$ & 0.5 & 0.55 \\
$\sigma_{\Delta p / p}$ & $2.25 \times 10^{-4}$ & $6 \times 10^{-4}$ \\
\hline \hline
\end{tabular}

Reasons for Difference

Besides the obvious difference in bunch structure between coalesced and uncoalesced protons which we have described earlier, there are other differences because of the way they are formed in the Main Injector. We have summarised these differences in Table 4. 


\section{Evolution of $\xi_{h}$ at Injection Porch}

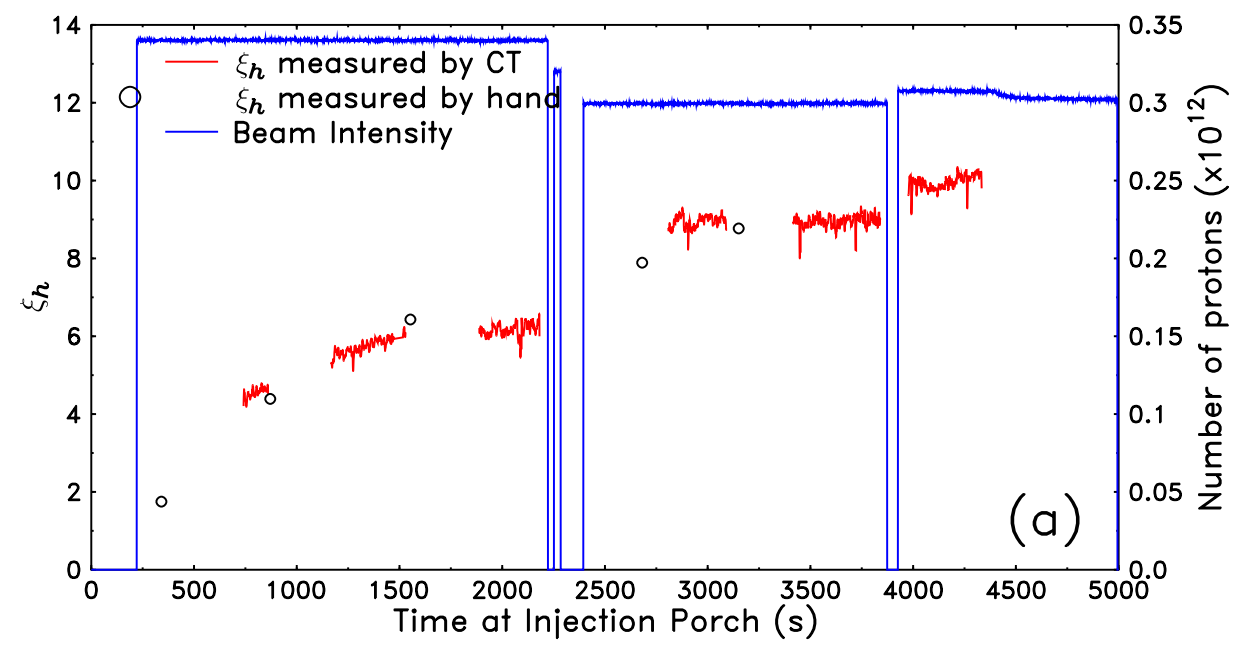

Evolution of $\xi_{h, v}$ at Injection Porch

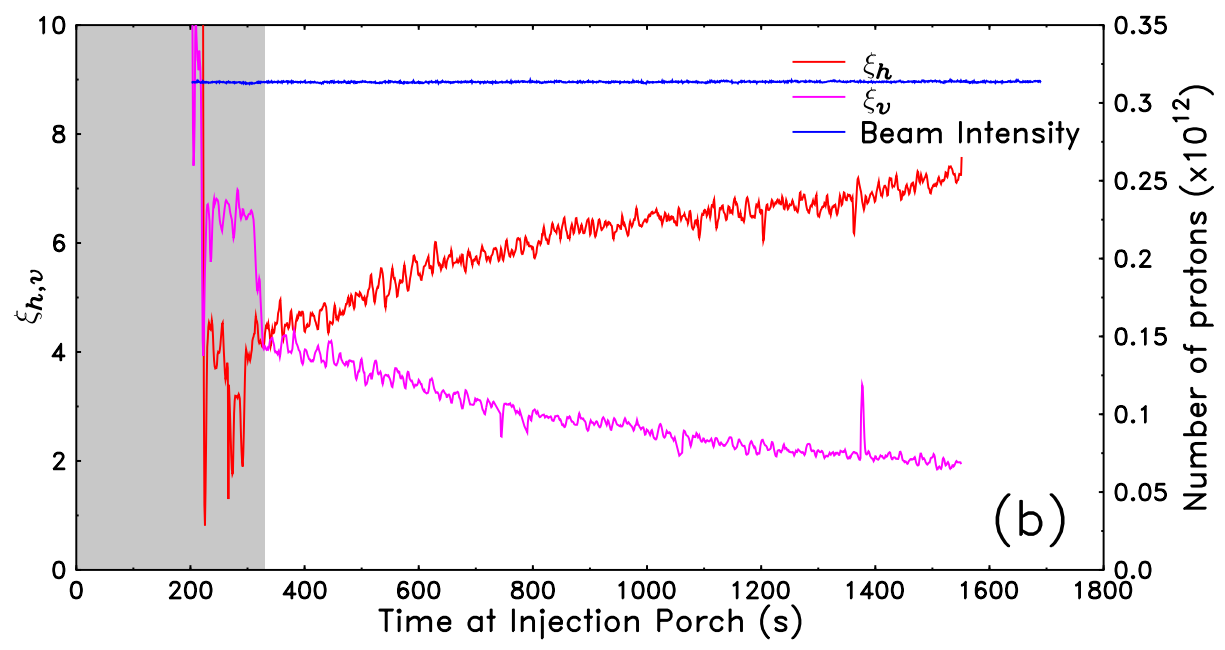

Figure 14 The CT tracks the chromaticity drift at the injection porch of the Tevatron after $15 \mathrm{~min}$ at low beta, unsqueezed and ramped down from high energy. It is interesting to notice in $(a)$ that after beam is reinjected at around $2500 \mathrm{~s}, \xi_{h}$ jumps from 6 units to 8 units with lower beam current. Grey area in $(b)$ is when $\mathrm{CT}$ is turned on and chromaticities is adjusted to 4 units. 
$\xi_{h, v}$ for Coalesced Beam

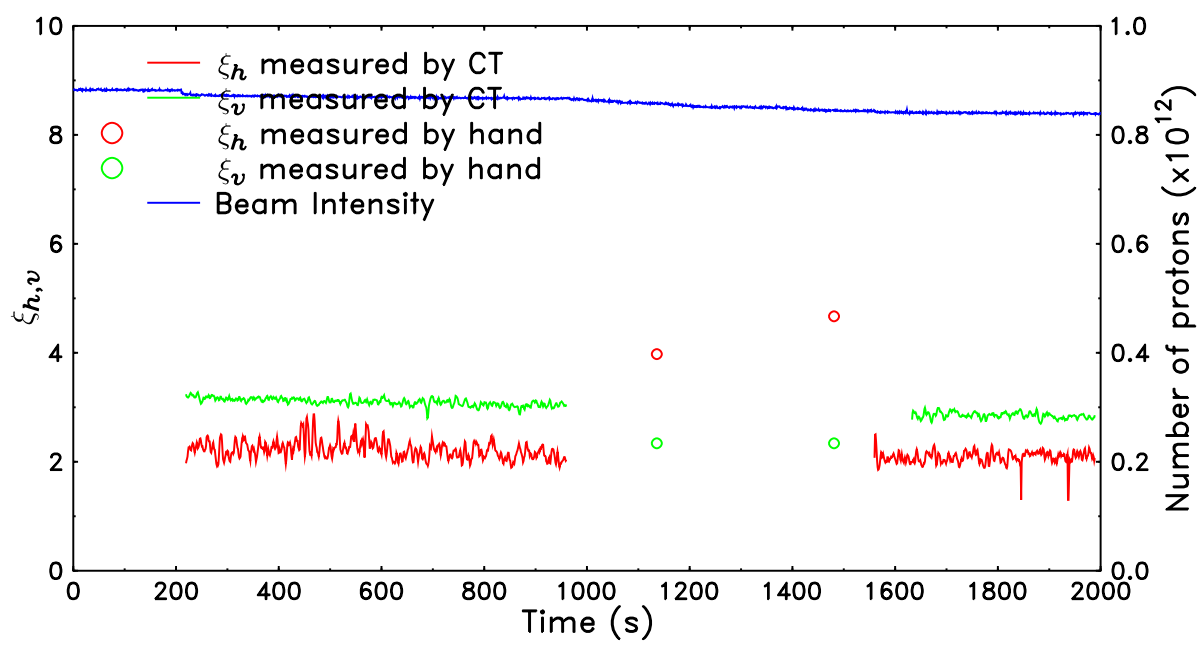

Figure 15 The CT measures $\xi_{h} \approx 2$ units and $\xi_{v} \approx 3$ units while measurements by the traditional method gives $\xi_{h} \approx 4$ to 5 units and $\xi_{v}=2.3$ units.

(Note: We have quoted beam size rather than emittance because it is sigma that is directly measured by the flying wires, optical transition radiation devices, and ionisation profile monitors in the Tevatron.) The transverse beam size quoted in this table is when the beam is at the Schottky detectors at A17. ${ }^{6}$ It is clear from this table that the horizontal beam size and the momentum spread are the two major differences between coalesced and uncoalesced beam. When we simulate the CT with the algorithm described in Appendix I and the parameters from Table 5, we find that it is, in fact, the transverse beam size (and surprisingly not momentum spread) that is the main cause of the discrepancy. Note: the simulation does not include dispersion but does include momentum spread. See Figure 16. From the simulation, we see that there is a $1 \mathrm{~mm}$ threshold where if the beam size is smaller than this, the chromaticity is essentially unaffected by beam size. However, if it is larger than $1 \mathrm{~mm}$, the measured chromaticity starts decreasing. This is consistent with the observation that the measured horizontal chromaticity of coalesced beam is smaller 
because its horizontal beam size is larger than $1 \mathrm{~mm}$. In fact, we have seen this effect in an experiment. See Figure 17(a). The chromaticity takes a step decrement after each ping of uncoalesced beam because the emittance and thus the beam size is increased. The horizontal betatron tune $Q_{h}$ also decrements after every ping, but we have checked that this is due to drops in chromaticity because when the chromaticity is changed, $Q_{h}$ also changes. See grey box in Figure 17(b). However, when $Q_{h}$ is changed, $\xi_{h}$ remains constant. This experiment shows that it is chromaticity which causes the betatron tune change and not vice versa.

For the vertical, the discrepancy should be smaller or non-existent because the vertical size is comparable. However, we still see a discrepancy of about 1 unit and so beam size cannot be the entire answer.

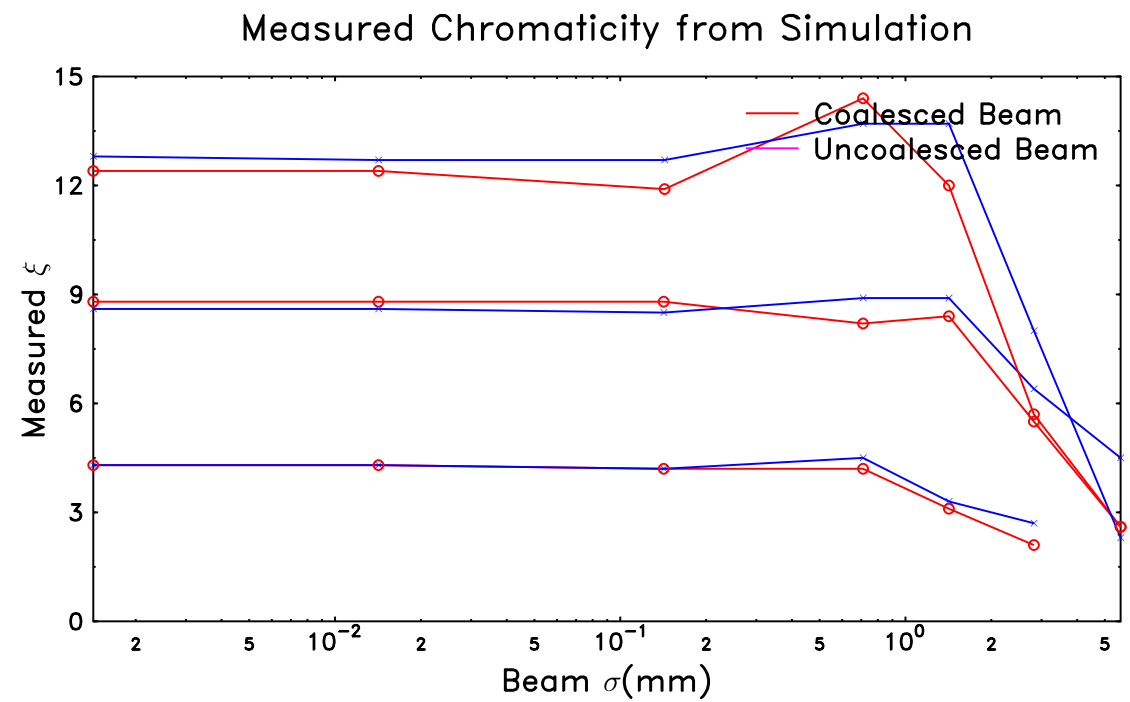

Figure 16 In the simulation, when the beam sigma is less than $1 \mathrm{~mm}$, the measured chromaticity is constant but when the beam sigma becomes larger than $1 \mathrm{~mm}$, the measured chromaticity descreases. Care has been taken in the simulation to ensure that the emittance does not blow up from the TT kick and the RF modulation and there is good $\mathrm{S} / \mathrm{N}$ for extracting the chromaticity. 


\section{Other Reasons}

Since beam size does not explain all the difference in measured chromaticity between uncoalesced and coalesced beam, we have empirical evidence that the beam distribution changes with the RF frequency because the phase response of the beam does not behave in the way we expect. The phase response is important ${ }^{1,3,4}$ because both the TT and the CT look at the null of the phase response. The TT tracks the phase null, while the CT essentially measures the distance of the phase null from its nominal position. Therefore, by measuring the phase response for different RF frequencies, we can see why the TT and CT measured chromaticities are smaller than we expect for coalesced beam.

For example, using coalesced beam for horizontal chromaticity $\xi_{h}=4$, we expect that the phase null should move by \pm 0.001 tune units for an RF frequency change $\Delta f=\mp 40 \mathrm{~Hz}$ and with the Tevatron parameters shown in Table 1. However, looking at Figure 18, we do not see this. The intersections of the red and blue circles in the figure shows where we expect the phase null of the response to be for $\Delta f=+40 \mathrm{~Hz}$ and $-40 \mathrm{~Hz}$ respectively. In fact, for $\Delta f=-40 \mathrm{~Hz}$, the null of the phase response does not go far enough to touch the intersection marked by the blue circle. This means that the measured chromaticity will be smaller than what we expect.

Our suspicion that the distribution has changed comes from observing that the slope

of the phase response in Figure 18. We notice that the slope for $\Delta f=-40 \mathrm{~Hz}$ about zero is steeper compared to the slope for $\Delta f=+40 \mathrm{~Hz}$. This is a strong indication that there is a transverse distribution change between these two RF frequency settings.

\section{CT Large Chromaticity Limitation}

For $\xi>10$, the CT does not measure the correct value despite having a clear carrier 
$\xi_{h}$ Decreases After Every Ping

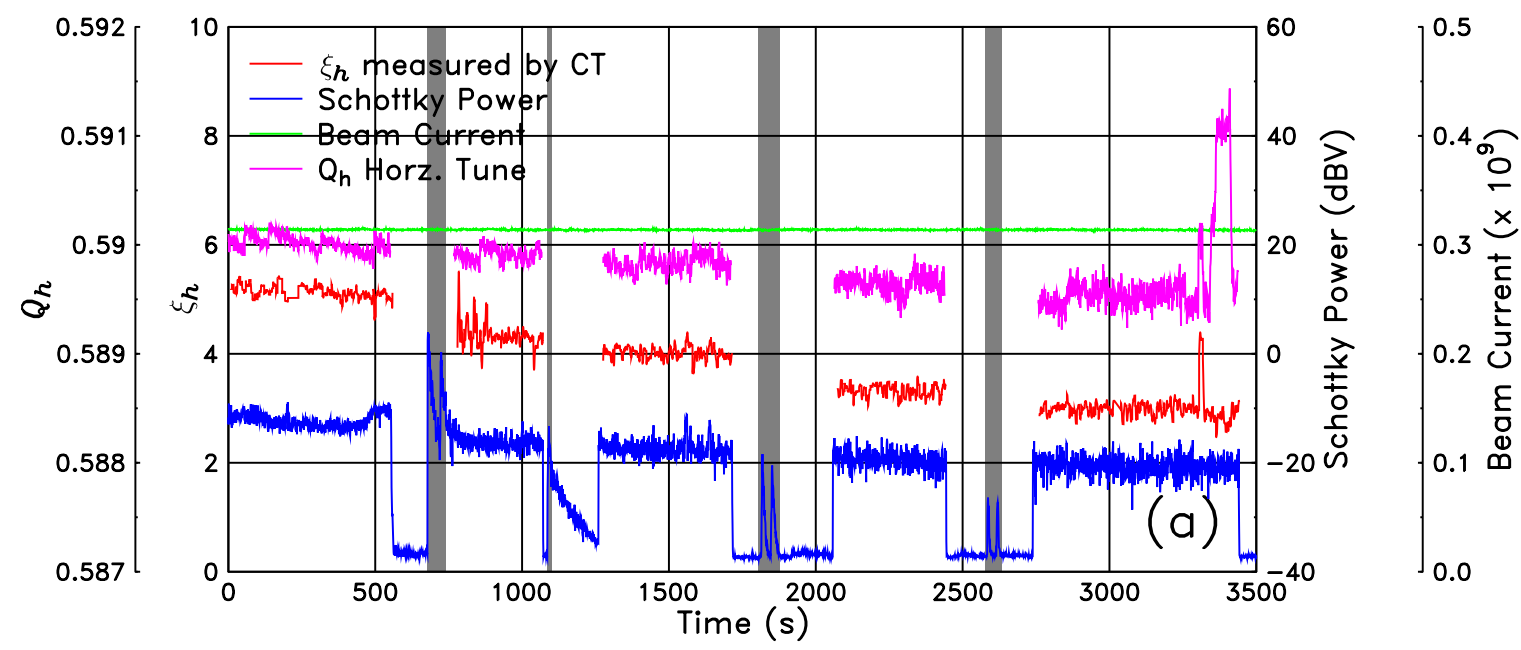

Zoomed in View

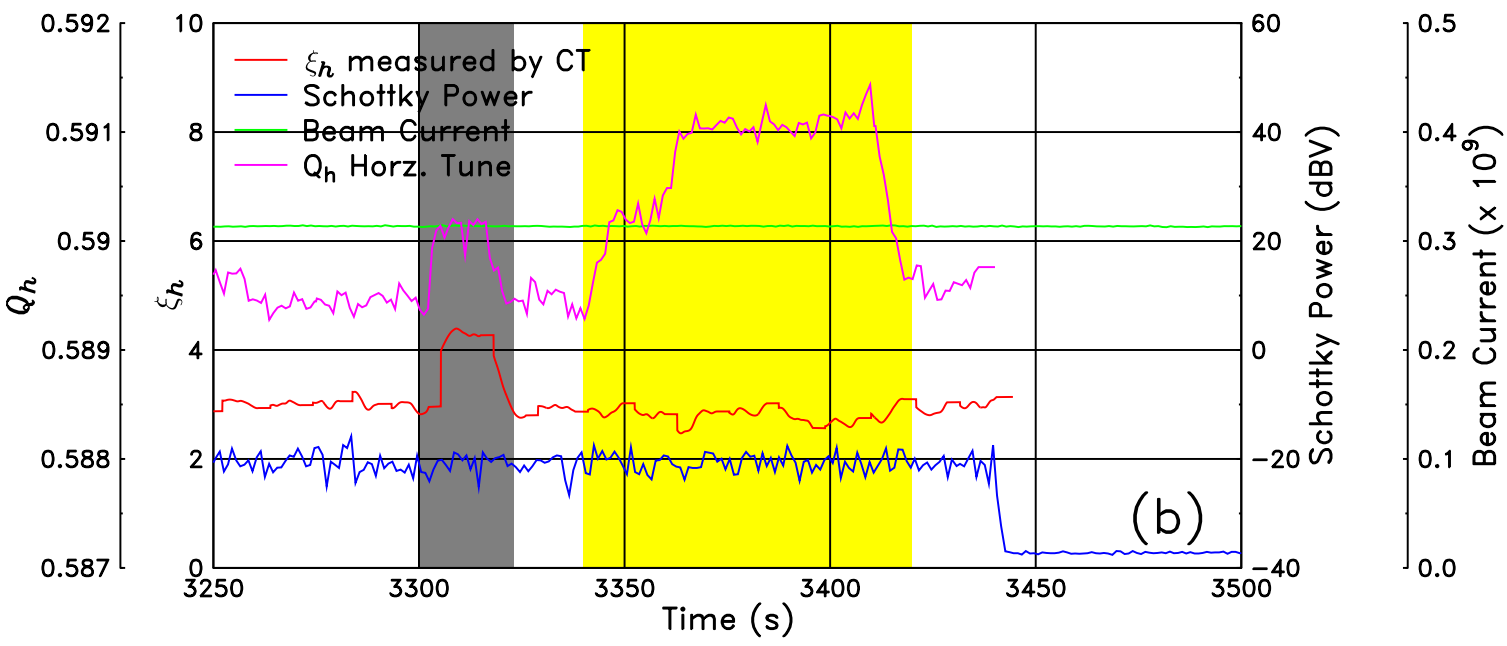

Figure 17 In (a) the measured chromaticity takes a discrete step down every time the beam is pinged. The sharp peaks (enclosed in grey) in the Schottky power is the moment when the pinger kicks the beam. The beam current is unaffected during this experiment. In (b), we zoom into 3250 to $3500 \mathrm{~s}$, when we change the chromaticity, the betatron tune changes (enclosed in grey), but when the betatron tune is changed the chromaticity remains constant (enclosed in yellow). 


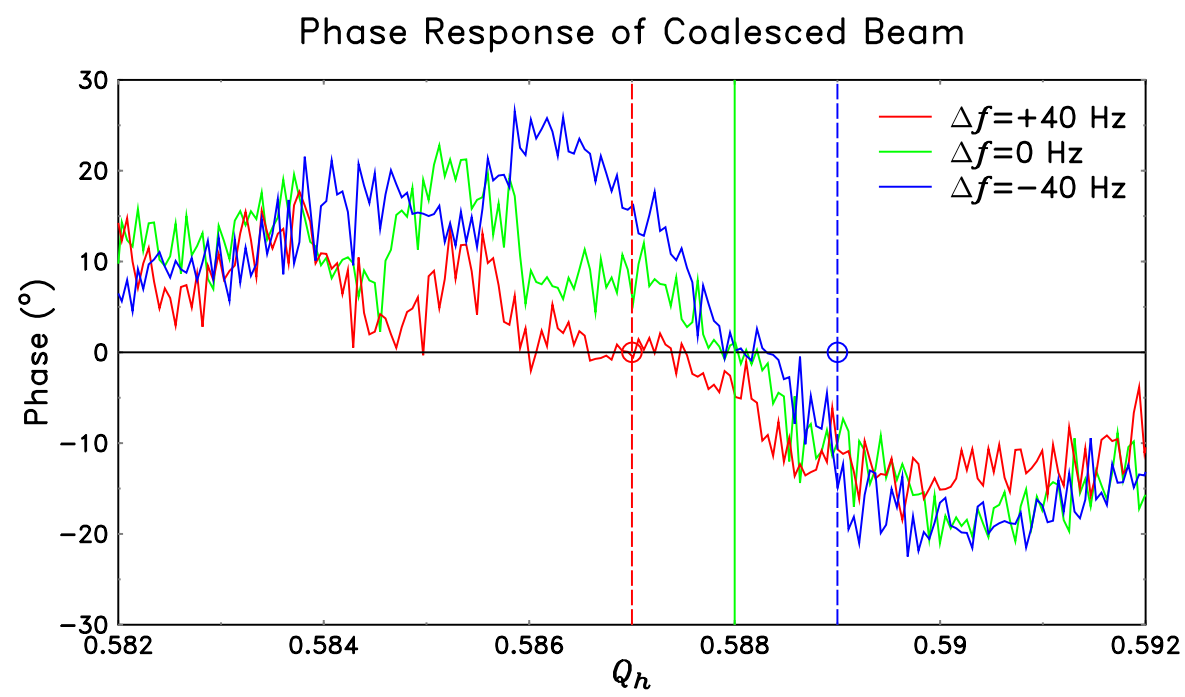

Figure 18 The phase response of coalesced beam. Notice that the null of the phase response does not move symetrically about $\Delta f=0$ when the RF frequency is changed from $-40 \mathrm{~Hz}$ to $+40 \mathrm{~Hz}$. In fact it does not move enough when $\Delta f=-40 \mathrm{~Hz}$ to touch the intersection of the blue dashed line and the phase equal to zero horizontal line marked by the blue circle.

frequency from the TT. See Figure 19. From computer simulations, we can see that large synchrotron amplitudes interfere with the phase demodulation. However, in order to get this interference in our simulation the beam size must be large as well. But because we do not see a beam size increase when the chromaticity is increased when we measure it with the flying wire system, we cannot fully explain why we have this limitation. 


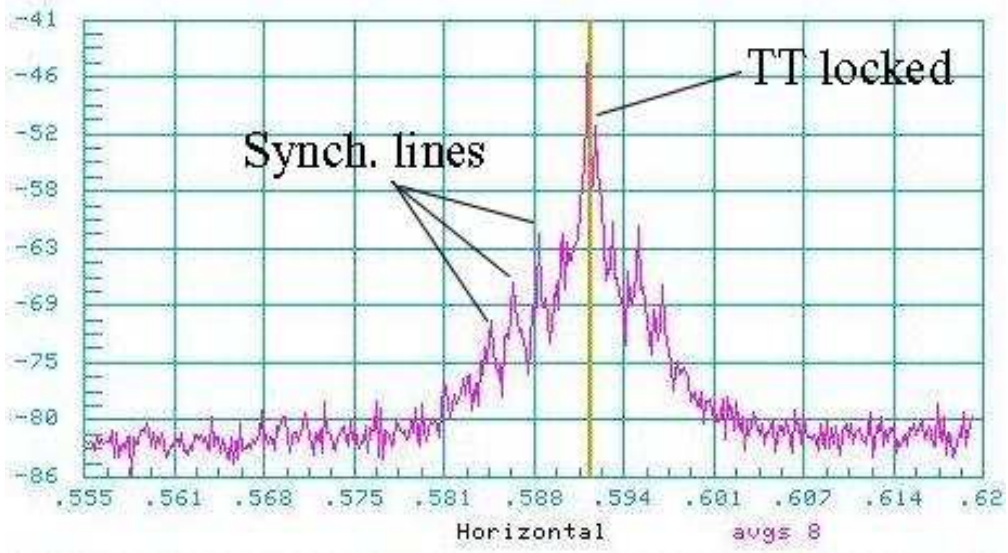

Figure 19 Shown here is the horizontal Schottky spectrum with the TT locked to the centre of the betatron tune distribution for $\xi_{h}=12$. The synchrotron lines are very distinct and large compared to the phase modulation lines, which, in fact, can hardly be seen at all. These large synchrotron lines interfere with the phase demodulation.

\begin{tabular}{c|c|l}
\hline \multicolumn{1}{c}{ Table 5. The Simulation Parameters } \\
\hline Parameter & Value & \multicolumn{1}{|c}{ Description } \\
$\eta$ & 0.0029 & slip factor \\
$Q_{0}$ & 0.588 & fractional betatron tune \\
$h$ & 1113 & harmonic number \\
$E_{s}$ & $150 \mathrm{GeV}$ & energy of the synchronous particle \\
$\nu_{s}$ & 0.00185 & synchrotron tune \\
$Q_{k}$ & $Q_{0}$ & TT kick tune \\
$\theta_{k}$ & $10^{-9}-10^{-6}$ & TT kick strength chosen for no emittance growth \\
$k_{3}$ & $h \eta / \nu_{s}$ & octupole strength \\
$\Delta \phi_{\bmod }$ & $10^{\circ}$ & amplitude of phase modulation \\
$\Omega_{\bmod }$ & $2 \pi \times 23 \mathrm{~s}^{-1}$ & phase modulation frequency \\
$N_{p}$ & $10^{4}-10^{5}$ & number of particles \\
$T_{\text {sim }}$ & 243 & simulation time in synchrotron periods \\
\hline \hline
\end{tabular}




\section{CONCLUSION}

The CT has been operational since 22 Oct 2008 and has been used in conjunction with the traditional method for HEP (high energy physics) shot set up. The operation of the CT has been reliable and it will replace the hand measured traditional method for HEP tune ups soon. The problems which we have mentioned in this paper will need to be resolved and machine studies have been requested to study the frequency response of the beam and to get to the bottom of why the CT has these limitations.

\section{ACKNOWLEDGEMENTS}

The author wishes to thank

(i) K. Koch for laying out and assemblying the electronics of the CT.

(ii) V. Ranjbar of Tech-X Corp., for discussions to understand the underlying cause of the measured chromaticity discrepancies between coalesced and uncoalesced beam. 


\section{APPENDIX I}

The formula which couples longitudinal phase modulated motion to transverse phase modulated motion has been derived for $\delta$-function type beams in both the longitudinal and transverse planes by McGinnis. ${ }^{3}$ However, real beams have finite sizes and emittances and so in order to understand this type of beam, we have derived the equations of motion which can be used in a computer simulation.

Let us suppose that the phase of the RF is modulated by a sinusoid of the form

$$
\phi_{\text {mod }}(t)=\Delta \phi_{\text {mod }} \sin \Omega_{\text {mod }} t
$$

and the RF is also a sinusoid

$$
V_{\mathrm{RF}}=V \sin \left(\omega_{\mathrm{RF}} t+\phi_{\mathrm{mod}}(t)\right)
$$

where $\Delta \phi_{\text {mod }}$ is the peak of the phase modulation, $\Omega_{\text {mod }}$ is the frequency of the phase modulation, $V$ is the peak voltage of the RF and $\omega_{R F}$ is the frequency of the RF. Then the longitudinal part is easily derived from Edwards ${ }^{7}$ with the inclusion of the phase modulation terms. (These equations can be compared with those used by Huang ${ }^{8}$ )

$$
\begin{aligned}
\varphi_{n+1}= & \varphi_{n}+2 \pi h \eta\left(\frac{\Delta p}{p}\right)_{n+1}+\Delta \phi_{\bmod }\left\{\sin \left[2 \pi \nu_{\bmod } \sum_{k=1}^{n+1}\left[1+\eta\left(\frac{\Delta p}{p}\right)_{k}\right]\right]\right. \\
& \left.-\sin \left[2 \pi \nu_{\bmod } \sum_{k=1}^{n}\left[1+\eta\left(\frac{\Delta p}{p}\right)_{k}\right]\right]\right\} \\
= & \varphi_{n}+2 \pi h \eta\left(\frac{\Delta p}{p}\right)_{n+1}+\Delta \phi_{\bmod } \times 2 \cos \left(2 \pi \nu _ { \operatorname { m o d } } \left\{\sum_{k=1}^{n}\left[1+\eta\left(\frac{\Delta p}{p}\right)_{k}\right]\right.\right. \\
& \left.\left.+\frac{1}{2}\left[1+\eta\left(\frac{\Delta p}{p}\right)_{n+1}\right]\right\}\right) \sin \left(2 \pi \nu_{\bmod } \times \frac{1}{2}\left[1+\eta\left(\frac{\Delta p}{p}\right)_{n+1}\right]\right) \\
\left(\frac{\Delta p}{p}\right)_{n+1}= & \left(\frac{\Delta p}{p}\right)_{n}+\frac{e V}{\beta^{2} E_{s}} \sin \varphi_{n}
\end{aligned}
$$


where $\nu_{\text {mod }}=\Omega_{\bmod } / \omega_{\text {rev }}$ is the modulation tune, and $\omega_{\text {rev }}$ is the revolution frequency of the synchronous particle, $h=\omega_{\mathrm{RF}} / \omega_{\text {rev }}$ is the harmonic number, $\beta \approx 1$ is the relativistic beta, $e$ is the the electron charge, $E_{s}$ is the energy of the synchronous particle and $\pi$ is the synchronous phase, i.e. we are above transition. Note: Technically, there is no synchronous particle when we modulate the RF. However, what we do is to assume that there is a reference RF system which is not modulated and then calculate phases and $\Delta p / p$ w.r.t. the synchronous particle in this reference RF system.

The equations of motion for the transverse part at the pickup with Courant-Snyder parameters $\left(\alpha_{p}, \beta_{p}\right)$ are

$$
\begin{aligned}
\theta_{n+1} & =\theta_{n}+2 \pi\left\{Q_{0}\left[1+\eta\left(\frac{\Delta p}{p}\right)_{n}\right]+\xi\left(\frac{\Delta p}{p}\right)_{n}\right\} \\
x_{n+1} & =a_{0} \cos \theta_{n+1} \\
p_{n+1} & =\beta_{p} x_{n}^{\prime}+\alpha_{p} x_{n} \\
& =-a_{0} \sin \theta_{n+1}+\Theta_{k} \sin 2 \pi n Q_{k}-k_{3} x_{n+1}^{3}
\end{aligned}
$$

where $\left(a_{0}, \theta_{0}\right)$ is the polar coordinate of the particle in normalised transverse phase space at $n=0, Q_{0}$ is the unperturbed betatron tune for the particle with zero $\Delta p / p$. There is a transverse kick from the TT which gives an angular kick of size $\Theta_{k}$ at the tune $Q_{k}$ and from an octupole with strength $k_{3}$.

First line of Equation (19)

For pedantic reasons, we will show how the betatron tune shift in the first line of (19) has been derived. From the definition of the betatron tune $Q$

$$
Q=\dot{\phi}_{Q} / \dot{\phi}_{\mathrm{rev}}
$$


we can write down the approximations to $\dot{\phi}_{Q}$ and $\dot{\phi}_{\text {rev }}$

$$
\left.\begin{array}{rl}
\dot{\phi}_{Q} & =\omega_{Q 0}\left(1+\frac{\Delta \omega_{Q}}{\omega_{Q 0}}\right) \\
\dot{\phi}_{\mathrm{rev}} & =\omega_{\mathrm{rev}}\left(1+\frac{\Delta \omega_{\mathrm{rev}}}{\omega_{\mathrm{rev}}}\right)
\end{array}\right\}
$$

$\omega_{Q 0}$ is betatron frequency when the modulation is zero, $\Delta \omega_{Q} / \omega_{Q 0}$ is the relative change in betatron frequency, and $\Delta \omega_{\text {rev }} / \omega_{\text {rev }}$ is the relative change in revolution frequency. If we assume that $\Delta \omega_{Q} / \omega_{Q 0}, \Delta \omega_{\text {rev }} / \omega_{\text {rev }} \ll 1$, we have

$$
\left.\begin{array}{rl}
\frac{\dot{\phi}_{Q}}{\dot{\phi}_{\mathrm{rev}}} & =Q_{0}\left(1+\frac{\Delta \omega_{Q}}{\omega_{Q 0}}\right)\left(1-\frac{\Delta \omega_{\mathrm{rev}}}{\omega_{\mathrm{rev}}}\right) \\
& \approx Q_{0}\left(1-\frac{\Delta \omega_{\mathrm{rev}}}{\omega_{\mathrm{rev}}}+\frac{\Delta \omega_{Q}}{\omega_{Q 0}}\right)
\end{array}\right\}
$$

because $\omega_{Q 0} / \omega_{\text {rev }}=Q_{0}$.

If we use the following relationships which involve $\Delta p / p, \Delta \omega_{\text {rev }} / \omega_{0}$ and $\Delta \omega_{Q} / \omega_{Q 0}$,

$$
\left.\begin{array}{rl}
\frac{\Delta \omega_{\mathrm{rev}}}{\omega_{0}} & =-\eta\left(\frac{\Delta p}{p}\right) \\
\frac{\Delta \omega_{Q}}{\omega_{Q 0}} & =\frac{\Delta Q}{Q_{0}}=\xi\left(\frac{\Delta p}{p}\right)
\end{array}\right\}
$$

then (22) becomes

$$
Q=Q_{0}\left[1+\eta\left(\frac{\Delta p}{p}\right)\right]+\xi\left(\frac{\Delta p}{p}\right)
$$

which when the rhs is examined, we find that the change in betatron tune has two contributions. One comes purely from momentum spread (contained in [.]) and the other from chromaticity. 


\section{APPENDIX II}

\begin{tabular}{c|c|c|c}
\multicolumn{4}{c}{ Table 3. Hilbert Transformer Filter Coefficients } \\
\hline $\boldsymbol{i}$ & coefficient $[\boldsymbol{i}]$ & $\boldsymbol{i}$ & coefficient $[\boldsymbol{i}]$ \\
0 & 0.0124427 & 50 & -0.0124427 \\
1 & 0.0000000 & 49 & 0.0000000 \\
2 & 0.00900775 & 48 & -0.00900775 \\
3 & 0.0000000 & 47 & 0.0000000 \\
4 & 0.0122483 & 46 & -0.0122483 \\
5 & 0.0000000 & 45 & 0.0000000 \\
6 & 0.0162677 & 44 & -0.0162677 \\
7 & 0.0000000 & 43 & 0.0000000 \\
8 & 0.0212626 & 42 & -0.0212626 \\
9 & 0.0000000 & 41 & 0.0000000 \\
10 & 0.0275401 & 40 & -0.0275401 \\
11 & 0.0000000 & 39 & 0.0000000 \\
12 & 0.0355514 & 38 & -0.0355514 \\
13 & 0.0000000 & 37 & 0.0000000 \\
14 & 0.0461607 & 36 & -0.0461607 \\
15 & 0.0000000 & 35 & 0.0000000 \\
16 & 0.0608791 & 34 & -0.0608791 \\
17 & 0.0000000 & 33 & 0.0000000 \\
18 & 0.0831083 & 32 & -0.0831083 \\
19 & 0.0000000 & 31 & 0.0000000 \\
20 & 0.121635 & 30 & -0.121635 \\
21 & 0.0000000 & 29 & 0.0000000 \\
22 & 0.208752 & 28 & -0.208752 \\
23 & 0.0000000 & 27 & 0.0000000 \\
24 & 0.635464 & 26 & -0.635464 \\
25 & 0.0000000 & & \\
\hline & & & \\
\hline
\end{tabular}



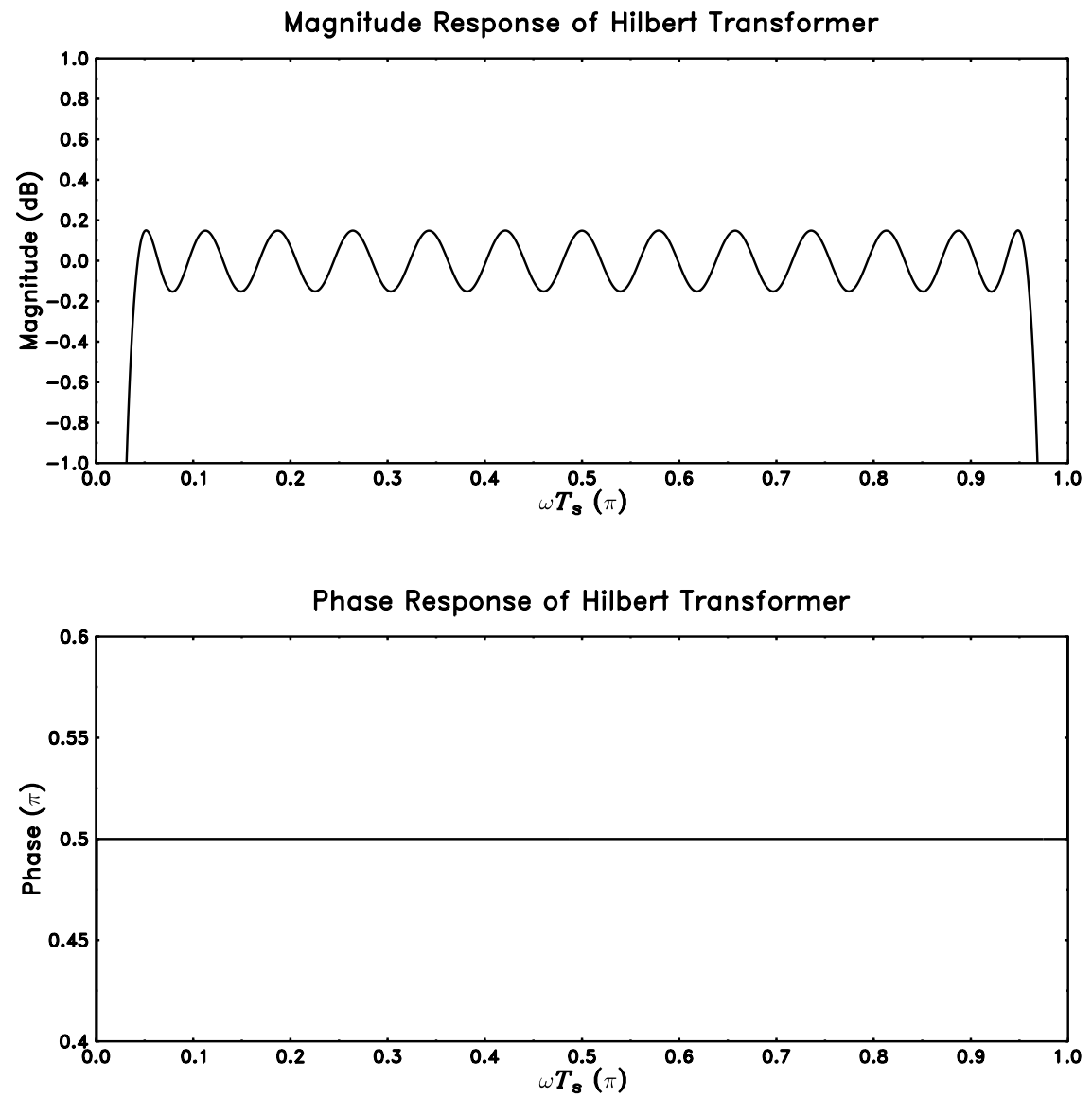

Figure 20 The frequency response of the Hilbert transformer using the coefficients shown in Table 3. 


\section{APPENDIX III}

The C code which implements an arctangent which knows about the winding number.

See $(12)$.

Listing $1 \mathrm{C}$ source listing for atan2unwind()

\#include <math.h>

$/ * * * * * * * * * * * * * * * * * * * * * * * * * * * * * * * * * * * * * * * * * * * * * * * * * * * * * * * * * * * * * * * * * * * * * *$

NAME

atan2unwind() -- Unwinds the phase given by atan2().

SYNOPSIS

atan2unwind() unwinds the phase given by atan2(). The range of $\operatorname{atan2()}$ is between $-p i$ and $p i$ and thus there is a discontinuity when the phase goes from the 3rd to the 4th quadrant and vice versa. Therefore, a phase unwinder is needed to smoothly change the phase at this transition. The simplest thing to do is to monitor the crossing between the 2nd and 3rd quadrant. If the phase goes from 3rd to 2nd, I increment the counter $n$, while if the phase goes from 2nd to 3rd I decrement the counter. For the other quadrants, I keep the counter $\mathrm{n}$ unchanged. The formula for calculating phase becomes:

$$
2 * n * M \_P I+\operatorname{atan} 2(y, x)
$$

NOTE: statics are used!!!!

USAGE

Call reset_atan2unwind() once before atan2unwind() is used to reset the global variables.

reset_atan2unwind() - reset the global variables.

atan2unwind (

$$
\begin{aligned}
& y-y \text { axis value of the phasor } \\
& \mathrm{x}-\mathrm{x} \text { axis value of the phasor }
\end{aligned}
$$

) - returns the unwound phase

AUTHOR

C.Y. Tan

SEE ALSO 
$\operatorname{atan} 2()$

LOGS

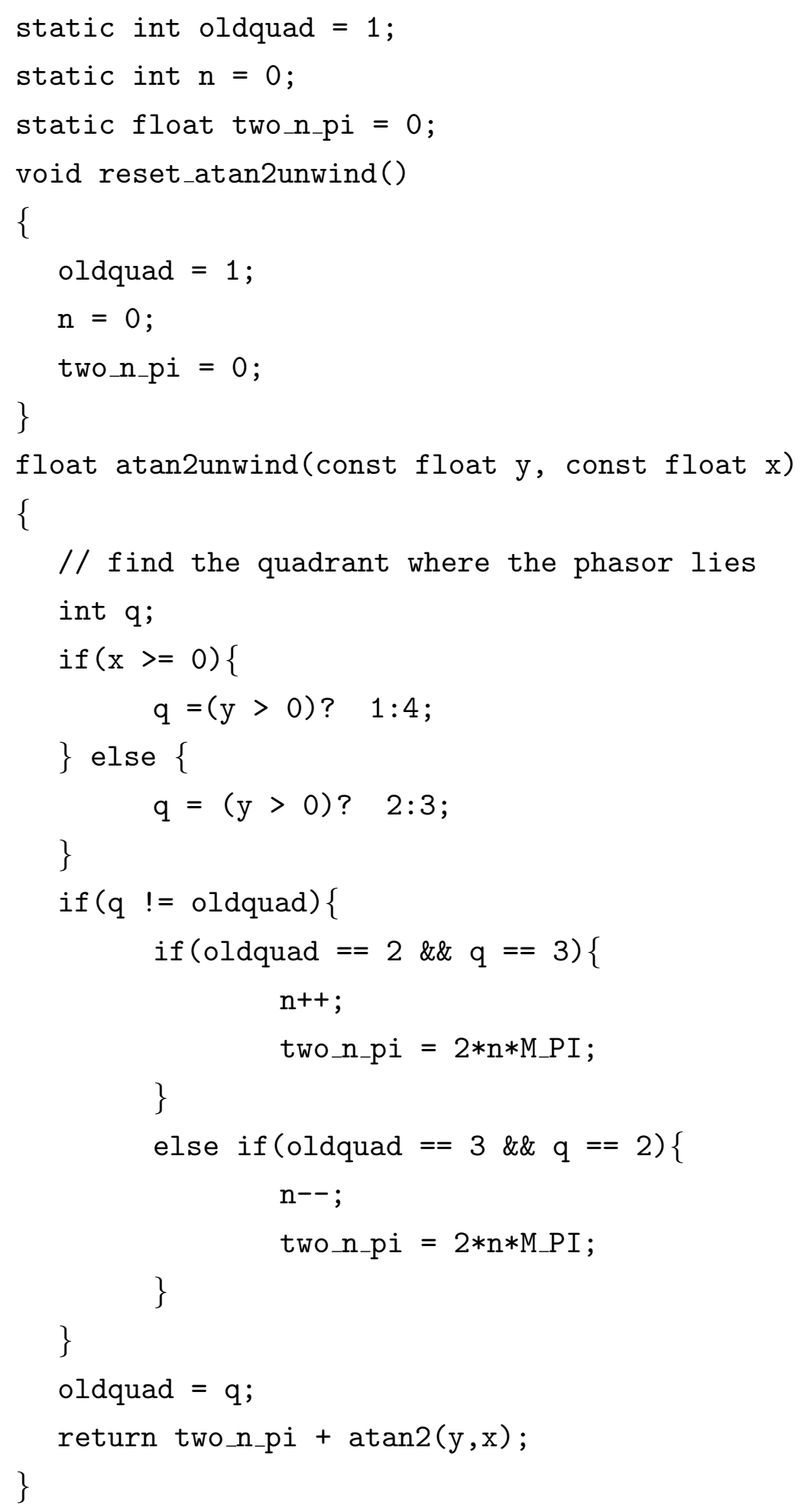




\section{REFERENCES}

[1] C.Y. Tan, The Tevatron Tune Tracker PLL - Theory, Implementation and Measurements, TM-2275, Fermilab 2004.

[2] C.Y. Tan and V. Ranjbar, Tevatron Electronic Log Book, 19 Mar 2005, 12 May 2005, 31 May 2005.

[3] D. McGinnis, Chromaticity Measurements using Phase Modulated RF and Vector Signal Analyzers, PBAR Note 656, Fermilab, 2001.

[4] C.Y. Tan, Preliminary Studies of a Chromaticity Tracker, TM-2346, Fermilab 2006.

[5] P. Bauer et al, Analysis of the B2 Correction in the Tevatron, Proc. PAC 2003, pg. $1730,2003$.

[6] V. Scarpine et al, Initial OTR Measurements of 150 GeV Protons in the Tevatron at FNAL, FERMILAB-CONF-06-103-AD, 2006.

[7] D.A. Edwards and M.J. Syphers, An Introduction to the Physics of High Energy Accelerators, pg. 34, John Wiley \& Sons, 1993.

[8] H. Huang et al, Experimental Determination of the Hamiltonian for Synchrotron Motion with RF Phase Modulation, Phys. Rev. E, 48, 4678 (1993). 